San Jose State University

SJSU ScholarWorks

Master's Theses

Master's Theses and Graduate Research

Summer 2021

\title{
Analyzing the Scalability of Parallel Microwire Arrays for Neural Recording
}

Yeena $\mathrm{Ng}$

San Jose State University

Follow this and additional works at: https://scholarworks.sjsu.edu/etd_theses

\section{Recommended Citation}

$\mathrm{Ng}$, Yeena, "Analyzing the Scalability of Parallel Microwire Arrays for Neural Recording" (2021). Master's Theses. 5209.

DOI: https://doi.org/10.31979/etd.5mf8-bst9

https://scholarworks.sjsu.edu/etd_theses/5209

This Thesis is brought to you for free and open access by the Master's Theses and Graduate Research at SJSU ScholarWorks. It has been accepted for inclusion in Master's Theses by an authorized administrator of SJSU ScholarWorks. For more information, please contact scholarworks@sjsu.edu. 


\title{
ANALYZING THE SCALABILITY OF PARALLEL MICROWIRE ARRAYS FOR NEURAL RECORDING
}

\author{
A Thesis \\ Presented to \\ The Faculty of the Department of Biomedical Engineering \\ San José State University \\ In Partial Fulfillment \\ of the Requirements for the Degree \\ Master of Science
}

by

Yeena $\mathrm{Ng}$

August 2021 
(C) 2021

Yeena $\mathrm{Ng}$

ALL RIGHTS RESERVED 
The Designated Reading Committee Approves the Project Titled

\title{
ANALYZING THE SCALABILITY OF PARALLEL MICROWIRE ARRAYS FOR NEURAL RECORDING
}

\author{
by \\ Yeena $\mathrm{Ng}$
}

APPROVED FOR THE DEPARTMENT OF BIOMEDICAL ENGINEERING

SAN JOSÉ STATE UNIVERSITY

August 2021

Guna Selvaduray, Ph.D. Department of Biomedical Engineering

Alessandro Bellofiore, Ph.D. Department of Biomedical Engineering

Yifan Kong, Ph.D. Chief Engineer, Paradromics, Inc. 


\section{ABSTRACT \\ ANALYZING THE SCALABILITY OF PARALLEL MICROWIRE ARRAYS FOR NEURAL RECORDING

\author{
by Yeena $\mathrm{Ng}$
}

Brain-computer interfaces (BCI) improve the quality of life for patients with severe motor disabilities and sensory impairment by providing them a direct way to communicate with the outside world through computers. To gain higher temporal resolution for better devices, intracortical neural electrodes, such as microwire arrays, are used. Microwire electrode arrays bonded to CMOS sensors, for intracortical neural recordings, have been claimed to be scalable. Microwire electrode arrays of varying diameters and densities were constructed and evaluated for percentage connectivity after interfacing with a custom-made CMOS sensor. The results demonstrate that there is no significant difference in the mean connectivity between a $3 \mathrm{~mm}$ and a $12 \mathrm{~mm}$ bundle as well as between arrays that have a wire-to-wire distance of $200 \mu \mathrm{m}$ versus $100 \mu \mathrm{m}$, confirming the scalability of microwire electrode arrays. Understanding array scalability allows for better electrodes to be built for higher resolution neural recordings, which can help those who suffer from motor or sensory disabilities regain a better quality of life by re-establishing some independence. 


\section{ACKNOWLEDGMENTS}

My work on this project could not have been done alone; I was supported by the guidance and mentorship of many individuals along the way, and I wish to express my gratitude to these individuals.

I am deeply grateful to my advisor, Professor Guna Selvaduray, for always believing in me and supporting me in my academic and professional endeavors. I am eternally grateful for all the guidance and support he provided me during my time at San Jose State University with my academics and my thesis. I could not have accomplished this without him.

I want to acknowledge all my coworkers at Paradromics, Inc., who worked on The Argo with me these past few years. Particularly, I wish to thank Yifan Kong, my mentor for the past three years, for teaching me invaluable skills and guiding me through my thesis writing. Mina Hanna, whose work I built upon and mentored me during my first year at Paradromics, and Harbi Sohal, who offered me wisdom and guidance with his data analysis expertise, also taught me useful skills and provided me with meaningful feedback. The following members of the hardware team were critical in the array development for the Argo System: Matt Hopper, Sashank Shivakumar, Aleksander Tadic, Bryan Kerr, Ryan Betzold, Kierra Boyle, and Kevin Boergens. Margo Straka and Kunal Sahasrabuddhe provided me with unending support throughout this process for which I am extremely grateful. Thank you to our CEO, Matt Angle, who has graciously allowed me to complete my thesis in conjunction with my work. Thanks to the unwavering 
support of every person in this company, I was able to complete this project and my degree.

In addition, I would like to thank all the faculty at San Jose State University who helped me through my master's degree, especially Professor Alessandro Bellofiore for being on my committee and providing feedback on my work. I would not have the experience I do today if it were not for the opportunities the Biomedical Engineering program at San Jose State University provided for me. 


\section{TABLE OF CONTENTS}

LIST OF TABLES......................................................... ix

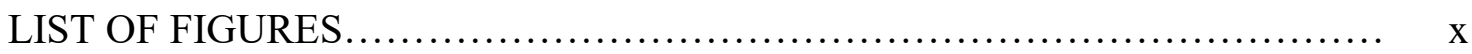

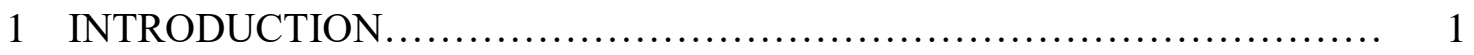

1.1 Brain-Computer Interfaces........................................ 1

1.2 Electrophysiology Modalities and Challenges.......................... 1

1.3 Refining Intracortical Neuron Recording Devices....................... 2

2 LITERATURE REVIEW ................................................ 4

2.1 Intracortical Neural Electrodes...................................... 4

2.2 Factors to Consider for Electrode Design............................. 4

2.2.1 Insertion Mechanics............................................ 4

2.2.2 Tissue Damage............................................ 5

2.2.3 Materials Selection............................................. 6

2.3 Current Array Designs................................................ 7

2.3.1 Utah Array.................................................... 7

2.3.2 Michigan Probe................................................ 8

2.3.3 Microwire Arrays............................................. 9

2.3.4 The Argo System............................................. 11

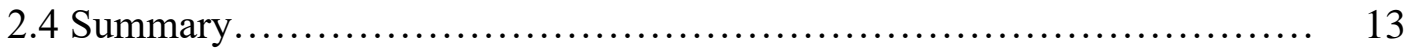

3 RESEARCH OBJECTIVES............................................. 15

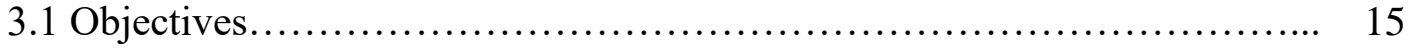

3.2 Justification.................................................... 15

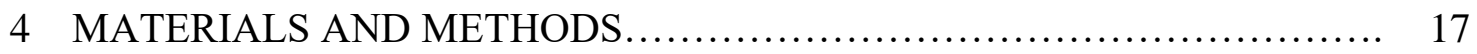

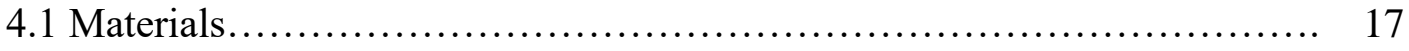

4.2 Methods....................................................... 18

4.2.1 Microwire Electrode Array Fabrication............................ 19

4.2.2 Connecting to a Sensor..................................... 23

4.2.3 Saline Bath Test............................................ 24

4.2.4 Calculating Percentage Connectivity.......................... 26

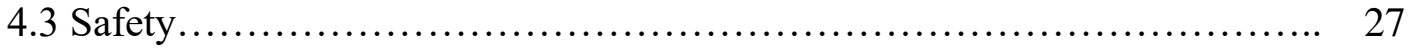

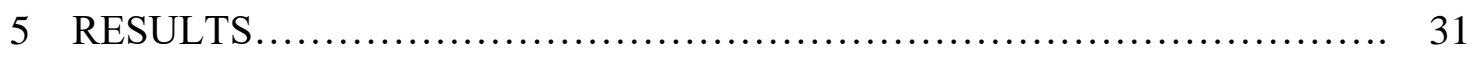

5.1 Array Diameter................................................... 32

5.1.1 Parametric Tests............................................ 32

5.1 .2 Non-parametric Tests...................................... 34

5.2 Electrode Density............................................. 35

5.2.1 Parametric Tests......................................... 35

5.2.2 Non-parametric Tests.................................... 38 


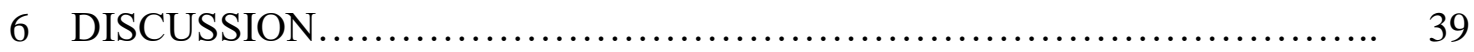

6.1 Array Diameter................................................... 39

6.2 Electrode Density............................................... 40

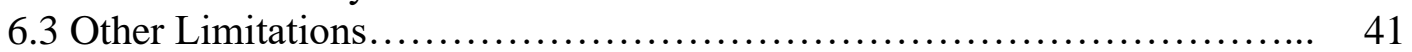

7 RECOMMENDATIONS FOR FUTURE RESEARCH.................... 43

7.1 Design of Experiments............................................ 43

7.1.1 Research Question and Hypothesis.............................. 43

7.1.2 Experimental Design..................................... 44

7.2 Statistical Analysis............................................... 45

7.2.1 Two-way Analysis of Variance............................... 45

7.2.2 Regression Model............................................ 46

7.2 .3 Error Analysis.................................................. 46

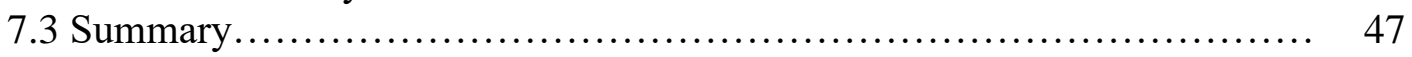

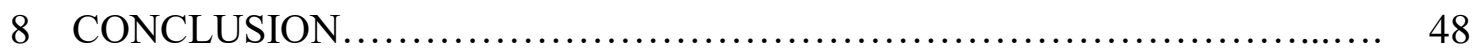

REFERENCES........................................................ 49

APPENDIX A: RAW PERCENTAGE CONNECTIVITY DATA................ 54 


\section{LIST OF TABLES}

Table 1. Comparison of the resolution and risk for varying electrophysiology

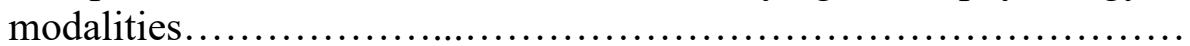

Table 2. Dielectric Constants of Common Insulators for Microelectrode

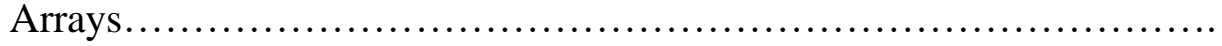

Table 3. Combinations of array sizes and electrode densities evaluated........... 18

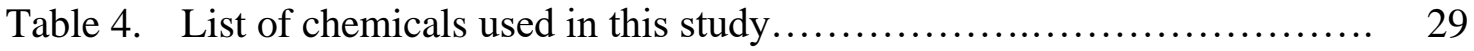

Table 5. Results of a one-way Analysis of Variance (ANOVA) ................. 34

Table 6. Results of a one-way Analysis of Variance (ANOVA) .................. 37

Table 7. Summary of Factors and Their Levels........................... 44 


\section{LIST OF FIGURES}

Figure 1. Photograph of a Utah Intracortical Electrode Array................. 8

Figure 2. Image of a Michigan microelectrode system, or Michigan probe, with lithographically-modified recording sites on the surface of the silicon planar shank

Figure 3. Fabrication procedure for microwire bundles, where $t$ represents the adjustable thickness of the sacrificial polymeric coating and $2 \mathrm{t}$ represents the center-to-center, or pitch, between each microwire (18)

Figure 4. A cross-section view of hexagonally-packed microwires in a bundle. The gold circles represent the metallic core of the microwire and the pale blue surrounding rings represent the sacrificial polymeric coating.

Figure 5. The Argo System utilizing fabricated microwire electrode arrays from Sahasrabuddhe et al., 2021

Figure 6. A microwire array secured onto the front plate of the Argo System headstage and held onto the CMOS sensor array with a flexible diaphragm.

Figure 7. Assembled microwire arrays after electrodes were coated with spatial Parylene-C coating and assembled in a clear heat shrink.

Figure 8. Proximal end of a $12 \mathrm{~mm}$ diameter, $200 \mu \mathrm{m}$ pitch bundle polished and with a small length of exposed wire prior for pressing onto a CMOS chip.

Figure 9. A fully fabricated microwire electrode array threaded onto the front plate ${ }^{(32)}$

Figure 10. (A) False color image of an electrochemically sharpened microwire taken with a scanning electron microscope (B) Image of insulated microwire array taken with a scanning electron microscope ${ }^{(32)}$......

Figure 11. Microwire bundle array secured in vise during pressing onto CMOS sensor. 
Figure 12. Example of a connectivity mask of a $3 \mathrm{~mm}$ diameter, $200 \mu \mathrm{m}$ pitch bundle showing final connected wires and pixels after filtering for gain and noise.

Figure 13. Example image of the proximal end of a $200 \mu \mathrm{m}$ array used to verify the number of wires within an array ............................ 26

Figure 14. Normal probability plot of data points in the sample............... 32

Figure 15. Box and whiskers plot showing the median, quartiles, and range of percentage connectivity of $3 \mathrm{~mm}$ and $12 \mathrm{~mm}$ diameter arrays..........

Figure 16. Percentile bootstrapping of the difference between means of $3 \mathrm{~mm}$ and $12 \mathrm{~mm}$ diameter bundles......................................

Figure 17. Box and whiskers plot showing the median, quartiles, and range of percentage connectivity of $200 \mu \mathrm{m}$ and $100 \mu \mathrm{m}$ pitch arrays...........

Figure 18. Percentile bootstrapping of the difference between means of $200 \mu \mathrm{m}$ and $100 \mu \mathrm{m}$ pitch arrays......................................... 


\section{INTRODUCTION}

\subsection{Brain-Computer Interfaces}

Patients who suffer from disabilities as a result of lack of neural function are missing a channel to connect them to the outside world. Neurological disorders or injuries that break down communication between the brain and the external world can cause severe sensory or motor impairment. One way to assist these patients in regaining their independence is through brain-computer interfaces (BCI), devices that connect the human brain to a machine that allows patients with limited muscular movements or other disabilities to communicate directly with the external world. BCIs utilize electrodes to record and relay information on neural activity back to a machine. The machine is then able to process and analyze these action potentials. Ultimately, BCI systems that can record and apply potentials have the potential to substantially improve the quality of life for patients by restoring some of their motor abilities, thus allowing them to live more independently ${ }^{(1)}$.

\subsection{Electrophysiology Modalities and Challenges}

Modern neural electrophysiological technologies optimize for less invasiveness and higher signal resolution. Non-invasive modalities, such as electroencephalography (EEG), record neural activity over the surface of the scalp and have been successful in

performing basic tasks on neuroprostheses and wheelchairs ${ }^{(2,3)}$. However, EEG lacks the resolution to perform more advanced tasks, such as multi-dimensional control applications especially in neuroprostheses, and more invasive approaches would be required to gain signal resolution ${ }^{(2,3,4)}$. 
More invasive approaches involve surgical interventions that pose far greater health risks but reward with higher spatial and temporal resolution gained from directly interfacing with brain tissue. Electrocorticography $(\mathrm{ECoG})$ involves placing an electrode grid directly on the surface of the brain ${ }^{(4)}$. ECoG can record from either directly on top of the dura or beneath the dura, providing substantially higher signal quality than EEG ${ }^{(5)}$. Studies have demonstrated that ECoG signals can be used to decode two-dimensional joystick trajectories in humans ${ }^{(6)}$.

Alternatively, intracortical neuron recording involves using penetrating electrodes to record from inside the cortex for the highest resolution, as these electrodes can come closest to firing neurons. As shown in Table 1, intracortical neuron recording demonstrates better temporal and spatial resolution than other methods. Electrodes implanted into the cortex can detect the spiking activity of single neurons, resolution unable to be obtained with the other modalities ${ }^{(4)}$.

Table 1. Comparison of the resolution and risk for varying electrophysiology modalities

\begin{tabular}{|c|c|c|c|}
\hline Modality & $\begin{array}{c}\text { Temporal } \\
\text { Resolution }\end{array}$ & Spatial Resolution & Risk \\
\hline EEG & $\sim 0.05 \mathrm{~s}$ & $\sim 10 \mathrm{~mm}$ & Non-invasive \\
\hline ECoG & $\sim 0.003 \mathrm{~s}$ & $\sim 5 \mathrm{~mm}$ & $\begin{array}{c}\text { Invasive (on top of } \\
\text { the dura or sub- } \\
\text { dura) }\end{array}$ \\
\hline $\begin{array}{c}\text { Intracortical } \\
\text { Neuron Recording }\end{array}$ & $\sim 0.003 \mathrm{~s}$ & $\sim 0.05-0.5 \mathrm{~mm}$ & $\begin{array}{c}\text { Invasive } \\
\text { (intracortical) }\end{array}$ \\
\hline
\end{tabular}

\subsection{Refining Intracortical Neuron Recording Devices}

Intracortical neuron recording provides higher quality recordings than other modalities, making it a popular choice for studies ${ }^{(7,8,9,10,11,12)}$. Implanted electrodes 
record the activity of nearby neurons. Neural activity can be broadly separated into two categories: multi-unit activity (MUA) and single-unit activity (SUA) ${ }^{(13)}$. Multi-unit activity comprises recordings of many units within the proximity of the electrode, while single-unit activity is the activity of individual neurons. The capability to record SUA is highly sought after, as it provides substantially higher resolution than MUA ${ }^{(13,14)}$.

The Utah Intracortical Electrode Array, a 100-electrode silicon array, has been evaluated in prior studies, including chronic human trials, due to its ability to record single action potential rather than thousands ${ }^{(15,16)}$. However, there is interest in developing scalable microwire arrays that improve upon signal resolution by increasing the number of recording electrodes and reducing the damage upon penetration into the tissue ${ }^{(7,17,18)}$. Electrode arrays that are easily fabricated to increase in size and density can open the doors to the rapid development of substantially more sensitive devices. 


\section{LITERATURE REVIEW}

\subsection{Intracortical Neural Electrodes}

Intracortical neuron recording utilizes implanted electrodes to record neural activity within the cortex of the brain. This modality of neural recording can be done with an array of micromachined electrodes, such as the Utah Array, a lithographically-modified

planar silicon shank, such as the Michigan probe, or microwire electrode arrays ${ }^{(10,15,18,19)}$. Electrodes must be optimized for easy scalability, penetration with minimal damage, and good signal-to-noise ratio ${ }^{(20)}$. However, other factors must be taken into consideration as well, such as insertion mechanics, tissue damage, and material selection.

\subsection{Factors to Consider for Electrode Design}

\subsubsection{Insertion Mechanics}

One of the primary factors to be considered while designing intracortical neuron recording electrodes is their insertion mechanics. Electrode insertion is a non-trivial procedure due to the robustness of the pia mater, a meningeal layer covering the brain $(22,22,23,24)$. Though flexible devices reduce chronic inflammation by minimizing material softness mismatch between the implant and the brain, they frequently have difficulties penetrating the pia ${ }^{(25)}$. While larger electrodes may seem more attractive for their mechanical strength, their large size can more substantially damage brain tissue, as described in Section 2.2.2 ${ }^{(9,26)}$. Probes must also penetrate the pia while avoiding dimpling caused by the large amount of force required to insert an electrode relative to the resistance of the underlying cortical tissue ${ }^{(18,21)}$. This dimpling is amplified in arrays of closely spaced electrodes, causing a bed-of-nails effect, deforming the brain tissue, and 
causing long-term damage ${ }^{(8,11,21,27,28)}$. Hence, it is prudent to consider how the shape and size of an electrode array affects its insertion capabilities.

\subsubsection{Tissue Damage}

Aside from insertion mechanics, tissue damage is also a critical factor to consider while designing intracortical neural electrodes. Probe insertion can rupture vasculature on the surface of the brain, leading to loss of blood to the damaged area of the brain ${ }^{(9)}$. Injury to the blood-brain barrier can also cause microglia and astrocytes to elicit an immune response, damaging the probe and reducing recording performance ${ }^{(9)}$. Microglial cells, as part of the immune response, encapsulate electrodes by forming a sheath, reducing ion and molecular exchange between the electrodes and the neurons (24). Furthermore, common materials utilized to build implants are susceptible to absorption of proteins, such as proinflammatory molecules and cytokines that perpetuate a chronic tissue response ${ }^{(25)}$. This encourages cellular encapsulation of the electrodes, reducing recording quality ${ }^{(25)}$.

Intracortical electrode arrays displace brain tissue volume when inserted, placing more pressure on surrounding tissue ${ }^{(25)}$. Furthermore, intracerebral hemorrhage edema cause swelling and pinching of nearby blood vessels, causing loss of perfusion and a secondary ischemic injury ${ }^{(25)}$. Aside from tissue displacement, strain caused by mechanical mismatch between the implanted device and the brain is a source of chronic tissue reaction due to micromotion, causing persistent irritation and prolonged inflammation ${ }^{(9,25)}$. 
Minimizing tissue damage is a non-trivial matter to consider for intracortical neuron recording modalities. Decreasing device size reduces tissue displacement and damage due to pressure but results in technical challenges to minimize feature sizes ${ }^{(25)}$. Other methods to reduce chronic damage due to micromotion include ways to decrease bending stiffness of the inserted electrodes ${ }^{(25)}$. Reducing material stiffness mismatch between brain tissue and the electrode by reducing the cross-sectional area or geometry of the device, increasing electrode length, and reducing the elastic modulus of the material have been proposed ${ }^{(25,29)}$. However, if the electrodes are too thin, they lack the mechanical strength to penetrate the brain ${ }^{(25)}$. Hence, further improvements regarding electrode size and shape must be made.

\subsubsection{Materials Selection}

The biocompatibility of materials for intracortical neuron recording electrodes is important to ensure the fidelity of long-term recordings. Intracortical neural electrode arrays are predominantly made from silicon-based microelectrodes due to their ease of reproduction, low costs of manufacturing, and customizability ${ }^{(30)}$. For example, the Utah array is a micromachined silicon-based array sputtered with an iridium oxide or platinum layer to improve conductivity at the electrode tips and insulated along its shaft with a biocompatible polymer parylene-C ${ }^{(31,32)}$. Similarly, the Michigan probe is built from a lithographically-modified planar silicon shank ${ }^{(19,31)}$. More details regarding these two common types of intracortical electrode arrays are discussed later, in Sections 2.3.1 and 


\subsection{2.}

Because of the manner in which microwire electrode arrays are produced, more materials have been explored. Microwire electrode arrays have typically been insulated with biocompatible materials, such as polyimide, parylene-C, Teflon, and quartz glass $(10,28)$. Insulating materials with higher dielectric constants are more favorable for minimizing stray capacitances from affecting the electrode during recording, as shown in Table 2; however, biocompatibility and ease of process development must also be taken into consideration $^{(7,23,29,30,33)}$. Conductive metallic cores of these microelectrodes have been made from biocompatible materials such as platinum, iridium, platinum-iridium, gold, stainless steel, tungsten, and molybdenum ${ }^{(30,35,36)}$.

Table 2. Dielectric Constants of Common Insulators for Microelectrode Arrays

\begin{tabular}{|c|c|}
\hline Insulation Material & $\begin{array}{c}\text { Dielectric Constant (1 kHz, Room } \\
\text { Temperature) }\end{array}$ \\
\hline Polyimide & 3.4 \\
\hline Parylene-C & 3.1 \\
\hline Teflon & 2.002 \\
\hline Quartz glass & 3.823 \\
\hline
\end{tabular}

Note: Dielectric constant values were sourced from several works. ${ }^{(34,35,36,37,38,39)}$

\subsection{Current Electrode Array Designs}

\subsubsection{Utah Array}

Currently, the two most used electrode designs are micromachined arrays and planar multi-electrode shanks. The Utah Intracortical Electrode Array is a 10 x 10 array of silicon probes spaced $400 \mu \mathrm{m}$ apart, center-to-center, as shown in Figure $1^{(15)}$. The 100 electrodes in the array are each $1.5 \mathrm{~mm}$ long and taper from $80 \mu \mathrm{m}$ to $50 \mu \mathrm{m}$ in tip diameter ${ }^{(15)}$. The Utah Array has been successfully utilized in neural prosthetic 
applications despite the low density of electrodes and limitations inherent to a $4 \mathrm{~mm} \mathrm{x}$ $4 \mathrm{~mm}$ cortical recording area ${ }^{(32,33)}$.

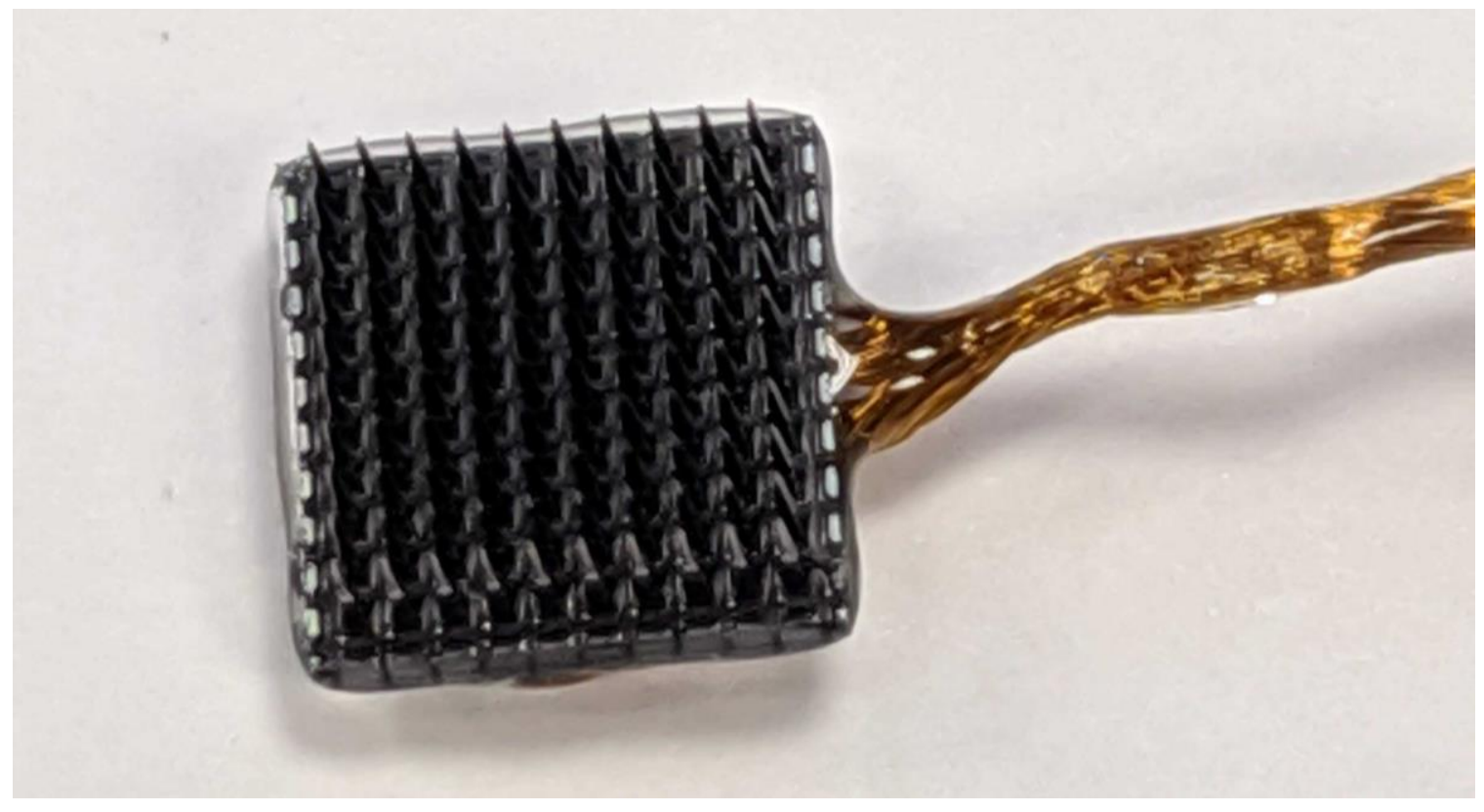

Figure 1. Photograph of a Utah Intracortical Electrode Array.

\subsubsection{Michigan Probe}

Another common form factor is a planar, shank-shaped electrode. The Michigan microelectrode system, or Michigan probe, is a silicon shank with lithographically patterned recording sites along the length of the substrate, allowing for electrophysiological information to be obtained at different depths of the neural cortex (19). This type of penetrating electrode, shown in Figure 2, can improve the resolution by scaling up the number of recording sites along the surface area of the flat part of the 10 $\mathrm{mm}$ long, $70 \mu \mathrm{m}$ wide, and $20 \mu \mathrm{m}$ thick substrate ${ }^{(31)}$. However, the number of recording sites is limited to the surface area of the electrode. 


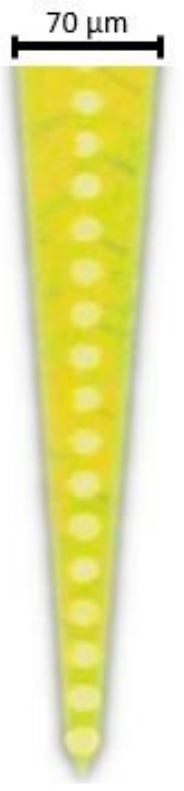

Figure 2. Image of a Michigan microelectrode system, or Michigan probe, with lithographically-modified recording sites on the surface of the silicon planar shank ${ }^{(21)}$.

\subsubsection{Microwire Electrode Arrays}

Neither of the two previously mentioned designs is easily scalable without major architectural changes, which raises the need for an alternative design. Microwire electrode arrays provide a possible solution for this problem. A microwire electrode array is built by arranging insulated wires into a bundle ${ }^{(7,17)}$. Unlike micromachined arrays, such as the Utah array, that has a fixed number of electrodes, the number of electrodes within a microwire bundle can be increased by increasing the diameter or density ${ }^{(18)}$.

Early microwire array bundles utilized less than 100 wires and penetrated the brain with the use of a metal carrier tube ${ }^{(7,12)}$. However, more recent arrays have demonstrated much greater scalability with microwire arrays. Obaid et al., 2020, built a $7 \mathrm{~mm}$ diameter 
array with $40 \mu \mathrm{m}$ wires at center-to-center distance, producing an 8640-electrode bundle, revealing the potential for microwire electrode array scalability ${ }^{(18)}$. This was produced with a fabrication process, shown in Figure 3, that bundled microwires by spacing wires with a sacrificial polymeric coating ${ }^{(18)}$. The sacrificial layer was customizable to adjust the center-to-center distance, or pitch, between the wires ${ }^{(18)}$. The coated electrodes were then formed into a round array by orienting the microwires in a hexagonal pattern, as shown in Figure $4^{(18)}$. This allows the array to theoretically be able to be scaled up to hundreds of thousands of microwires for high-bandwidth applications ${ }^{(18)}$.

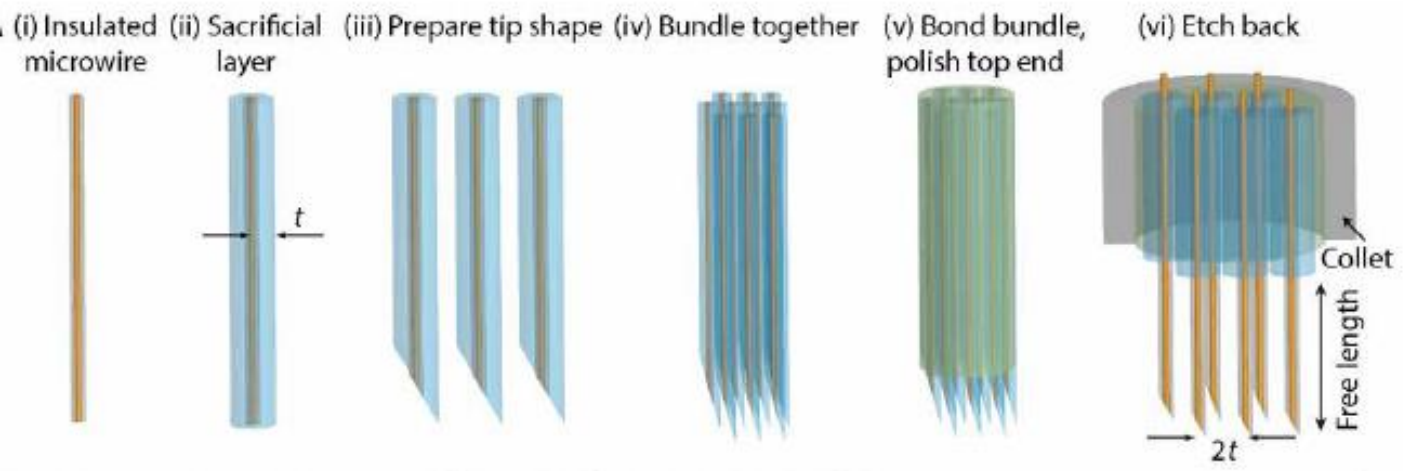

Figure 3. Fabrication procedure for microwire bundles, where $t$ represents the adjustable thickness of the sacrificial polymeric coating and $2 t$ represents the center-to-center, or pitch, between each microwire ${ }^{(18)}$. 


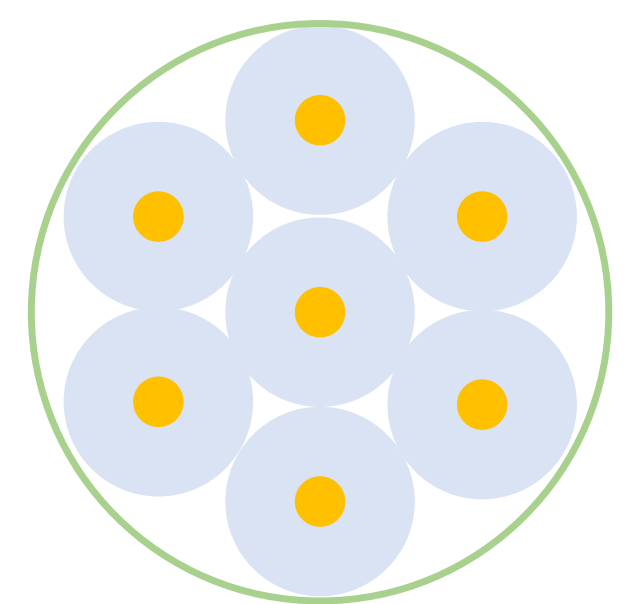

Figure 4. A cross-section view of hexagonally-packed microwires in a bundle. The gold circles represent the metallic core of the microwire and the pale blue surrounding rings represent the sacrificial polymeric coating.

In order to process the neural activity detected by the electrode array into meaningful information, the signal must amplified. The distal end of the array refers to the end that will be inserted into and recording from within the brain ${ }^{(18)}$. The proximal end of microwire electrode array is connected to the CMOS amplifier array by compressing the wires mechanically onto the chip, creating ohmic contacts ${ }^{(18)}$. This parallel bonding paradigm where the proximal end of the array is mated to CMOS electronics while the distal recording tips are inserted into the brain has been explored for many years ${ }^{(18,40)}$. This strategy worked well with many different CMOS designs, ensuring that more than $90 \%$ of wires contact reproducibly ${ }^{(18)}$.

\subsubsection{The Argo System}

In order to use microwire electrode arrays to record and decode neural activity, they must be paired with the appropriate electronics to read and amplify neural signals ${ }^{(10)}$. Previously, microwire electrodes were not considered as scalable recording methods due to difficulties connecting large arrays to many amplifiers ${ }^{(8,11)}$. However, recent successes 
in connecting microwire arrays to high density CMOS sensor arrays have proved that this problem could be resolved, demonstrating the viability of recording from substantially larger, denser microwire electrode arrays ${ }^{(18,40)}$. Sahasrabuddhe et al., 2021, describes the Argo System in Figure 5, where microwire bundles interface with a CMOS amplifier array to send recorded neural signals over an optical data link to the server computers ${ }^{(10)}$. The data are received by custom data acquisition software that displays it on a user interface on the client computer and saved into an offline processing computer for further analysis ${ }^{(10)}$.

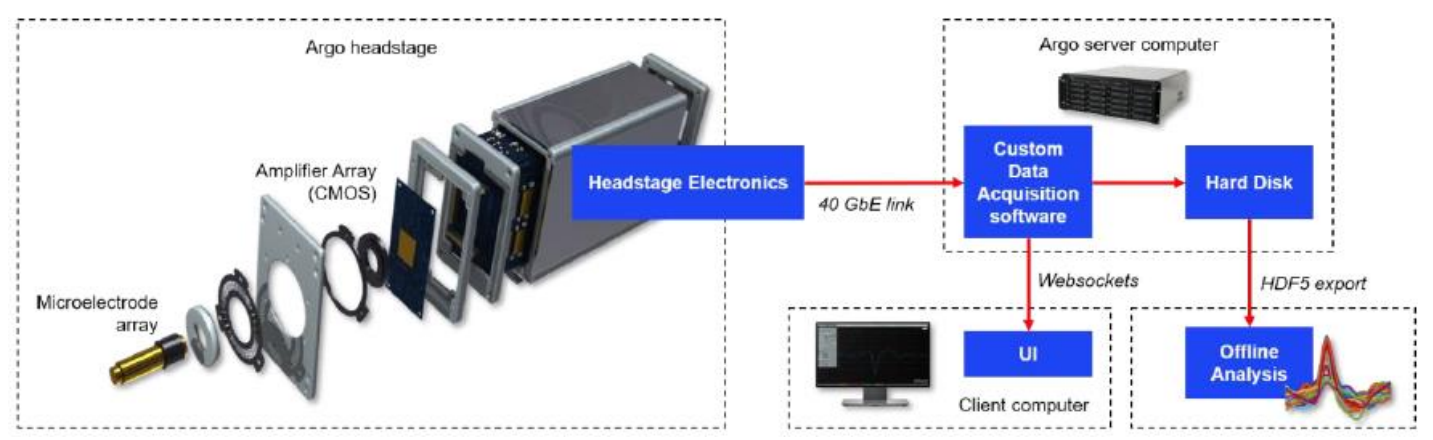

Figure 5. The Argo System utilizing fabricated microwire electrode arrays from Sahasrabuddhe et al., 2021.

The Argo System CMOS sensor, utilized to amplify and filter recorded signals from the electrode array, is an application-specific integrated circuit (ASIC) consisting of a $256 \times 256$ pixel array ${ }^{(10)}$. The pixels are spaced at $50 \mu \mathrm{m}$ pitch and contain a $40 \mu \mathrm{m} \times 40$ $\mu \mathrm{m}$ metal landing pad on top of each pixel to connect with the microwire electrodes ${ }^{(10)}$. Sahasrabuddhe et al., 2021, describes the chip design and relevant electronics in further detail. The microwire electrode bundle connects to the CMOS by mechanically pressing the distal end of the microwire array onto the chip, simultaneously contacting all the electrodes in the array with the pixels on the CMOS ${ }^{(10)}$. The bundle is held in place with 
a flexible diaphragm secured onto the front plate of the head stage of the Argo System as shown in Figure 6 and described by Obaid et al., $2020^{(18)}$. Successes seen with the Argo System make it, along with microwire electrode arrays, viable for recording neural activity.

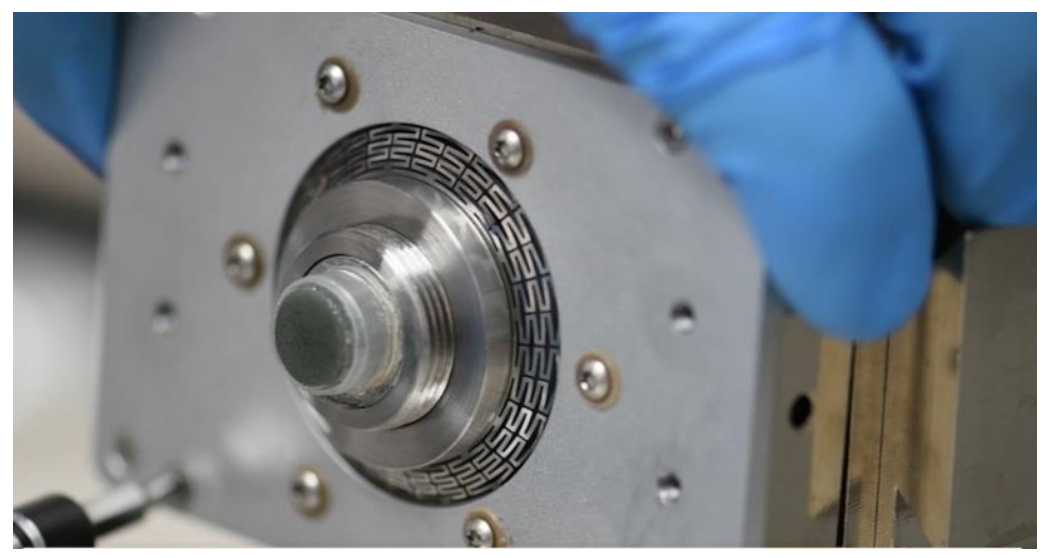

Figure 6. A microwire array secured onto the front plate of the Argo System head stage and held onto the CMOS sensor array with a flexible diaphragm.

\subsection{Summary}

Intracortical neuron recordings can capture neural signals with higher resolution, because the electrodes are substantially closer to the area of activity than other recording modalities. In order to develop electrodes, insertion mechanics, tissue damage, and materials selection must be taken into consideration. Microwire electrode arrays provide a scalable solution to these issues; the electrodes are much smaller in size than the traditional micromachined Utah Array, and microwire bundle architecture allows for scalability in terms of array size and density. Microwire electrodes also do not face the same surface area limitations as lithographically-modified planar shanks, such as the Michigan probe. Microwire electrode arrays, as demonstrated by the ones utilized in the Argo System, serve as viable intracortical neural recording devices that are easy to scale 
up in array size and electrode density. This opens doors for the development and manufacturing of high-resolution neural interfaces that can change the lives of patients with mobility impairment and sensory loss to potentially communicate directly with their electronics. This would provide patients with some independence as well as an improved quality of life. 


\section{RESEARCH OBJECTIVE}

\subsection{Objectives}

My research objective was to evaluate the scalability of microwire electrode bundles by using the aforementioned Argo System to measure electrode connectivity as a function of increasing array diameter and electrode density. I hypothesized that if the array diameter and electrode density increased, then the average connectivity will not be significantly different between larger, denser arrays and smaller, sparser arrays. Average connectivity refers to the number of wires connected to the CMOS sensor in the Argo System divided by the total number of electrodes in the bundle.

\subsection{Justification}

Electrode scalability for improved neural recording quality is a problem that can be resolved through microwire bundles. Microwires produce less damage upon insertion, due to their smaller size, than the traditionally micromachined arrays and the number of electrodes can be increased for easy scaling, unlike lithographically-modified planar shanks that are limited by the surface area of the probe. The fabrication method for these arrays, as described in Obaid et al., 2020, allows for an increase in size and density for use in different cortical areas for greater lateral coverage without implementing major changes to the manufacturing process. Increasing the array diameter can be done simply by incorporating more electrodes into the microwire bundle, and increasing the density merely involves reducing the thickness of the sacrificial spatial coating between each wire, allowing for more electrodes to be packed into the same volume. These small 
changes allow for simple customization of intracortical implants without drastically affecting the other steps in the manufacturing process

My hypothesis was that increasing the array diameter and electrode density would not cause a statistically significant change in the average connectivity of the electrode arrays. My null hypotheses were that arrays with $3 \mathrm{~mm}$ and $12 \mathrm{~mm}$ diameters would have the same mean percentage connectivity; similarly, arrays with $200 \mu \mathrm{m}$ and $100 \mu \mathrm{m}$ pitches would have the same mean percentage connectivity. My alternative hypotheses were that arrays with diameters of $3 \mathrm{~mm}$ and $12 \mathrm{~mm}$ diameter would have a statistically different mean percentage connectivity, and arrays with $200 \mu \mathrm{m}$ and $100 \mu \mathrm{m}$ pitches would also have a statistically significant difference in mean percentage connectivity. 


\section{MATERIALS AND METHODS}

\subsection{Materials}

The materials required to build the microwire array bundles included $18 \mu \mathrm{m}$ diameter platinum-iridium alloy (90\% platinum, 10\% iridium) wires purchased from TANAKA Precious Metals. Calcium chloride, deionized water, a programmable AC power source, a printed circuit board with a single strip of copper running from end to end, and silver epoxy H20E from Epoxy Technologies were used in the electrochemical sharpening process. A fume hood with carbon and HEPA filters was needed to electrochemically process the microwires. Parylene-C dimer DPX-C, Silane A-174, and Specialty Coating Systems PDS 2010 Labcoter were required to coat a spatial Parylene-C layer as well as the electrode insulation layer onto the wires. For the microwire array assembly, fluorinated ethylene propylene heat shrink tubing, a hot air gun, EPO-TEK 301 epoxy, a generic vacuum pump drying oven, and a custom 0.45 -inch long stainless steel tube with inner diameters between $1.5 \mathrm{~mm}$ to $2 \mathrm{~mm}$ were used. $96 \%$ sulfuric acid, $30 \%$ hydrogen peroxide, hot plates, beakers, and an acid hood were used to etch the epoxy. A plasma etcher (SPI Plasma Prep II) and pure oxygen gas were needed to etch spatial Parylene-C. To smooth out the top of the array, a 5C collet, lathe, and 400, 600, 1000, and 1200 grit silicon carbide grinding paper were needed. Fluorescent dye Y3G mixed with EPO-TEK 301 for contrast, a confocal microscope to measure the lasing distance, and a UV laser were used to ablate the insulation from the tips of the electrodes. A scanning electron microscope was critical for evaluation of the quality of recording sites 
on the tips of the electrodes. A Leica DVM6 digital microscope and ImageJ were used to assess the packing of the microwires within each array.

To assess the connectivity of the array, the Argo System as described in Sahasrabuddhe et al., 2021 and the custom CMOS chip which the electrode array mechanically presses onto were needed. The microwire arrays required custom hollowed 3/4"-20 threaded rods to thread into the front plates, cyanoacrylate, custom front plates, flexible diaphragms, custom stainless steel housing, and M2 x 0.4 screws to secure all the parts. A modified vise was required to press the electrode array onto the CMOS sensor. 1X PBS, a beaker, and a custom user interface were used to perform the saline test to evaluate the connectivity of the electrodes within the array to the CMOS chip ${ }^{(10)}$.

\subsection{Methods}

Understanding the effects of array diameter and electrode density of microelectrode arrays on the electrode array connectivity with the CMOS sensor results in better design decisions.

In order to do so, $3 \mathrm{~mm}$ and $12 \mathrm{~mm}$ diameters of microwire electrode arrays with low and high electrode densities were fabricated. Electrode densities were referenced by their "pitch," or distance from the center of one wire to the center of the next. Low-density arrays had a pitch of $200 \mu \mathrm{m}$, and high-density arrays had a pitch of $100 \mu \mathrm{m}$. Table 3 lists the combinations of array sizes and electrode pitches that were studied.

Table 3. Combinations of array sizes and electrode densities evaluated.

\begin{tabular}{|c|c|c|}
\hline Array Size & \multicolumn{2}{|c|}{ Electrode Pitch } \\
\hline $3 \mathrm{~mm}$ & $200 \mu \mathrm{m}$ (Low Density) & $100 \mu \mathrm{m}$ (High Density) \\
\hline $12 \mathrm{~mm}$ & $200 \mu \mathrm{m}$ (Low Density) & $100 \mu \mathrm{m}$ (High Density) \\
\hline
\end{tabular}


For additional clarification, the "proximal" end of the electrode array as referenced within this thesis refers to the end of the bundle mechanically pressed onto the CMOS chip. The "distal" end of the array refers to the end that will be recording from within the brain.

\subsubsection{Microwire Electrode Array Fabrication}

Microwire electrode arrays were fabricated from $18 \mu \mathrm{m}$ diameter platinum-iridium alloy (90\% platinum, 10\% iridium) wires purchased from TANAKA Precious Metals. The wires were annealed, cut into 1-inch long pieces, and one end of the wires was electrochemically processed in a $0.5 \mathrm{M}$ calcium chloride solution to produce a sharp needle-shaped electrode. The electrodes were coated with a spatial layer of polymer Parylene-C $(\mathrm{PaC})$ through chemical vapor deposition with a Specialty Coating Systems PDS 2010 Labcoter that determined the pitch between the wires. The coated wires were collected in a round piece of heat shrink and assembled into a round bundle with all the sharpened tips oriented in the same direction and on the same plane as shown in Figure 7. The electrodes were bound by epoxy EPO-TEK 301 from Epoxy Technology Inc and cured at $65^{\circ} \mathrm{C}$ for 2 hours. The array was then removed from the heat shrink and adhered with EPO-TEK 301 into a 0.45-inch long 316 stainless steel tube with inner diameters between $1.5 \mathrm{~mm}$ to $2 \mathrm{~mm}$ larger than the bundle to provide handling ease. The tube sat in the center of the bundle such that 0.25 inches of the distal end of the array extended beyond the edge of the tube. 


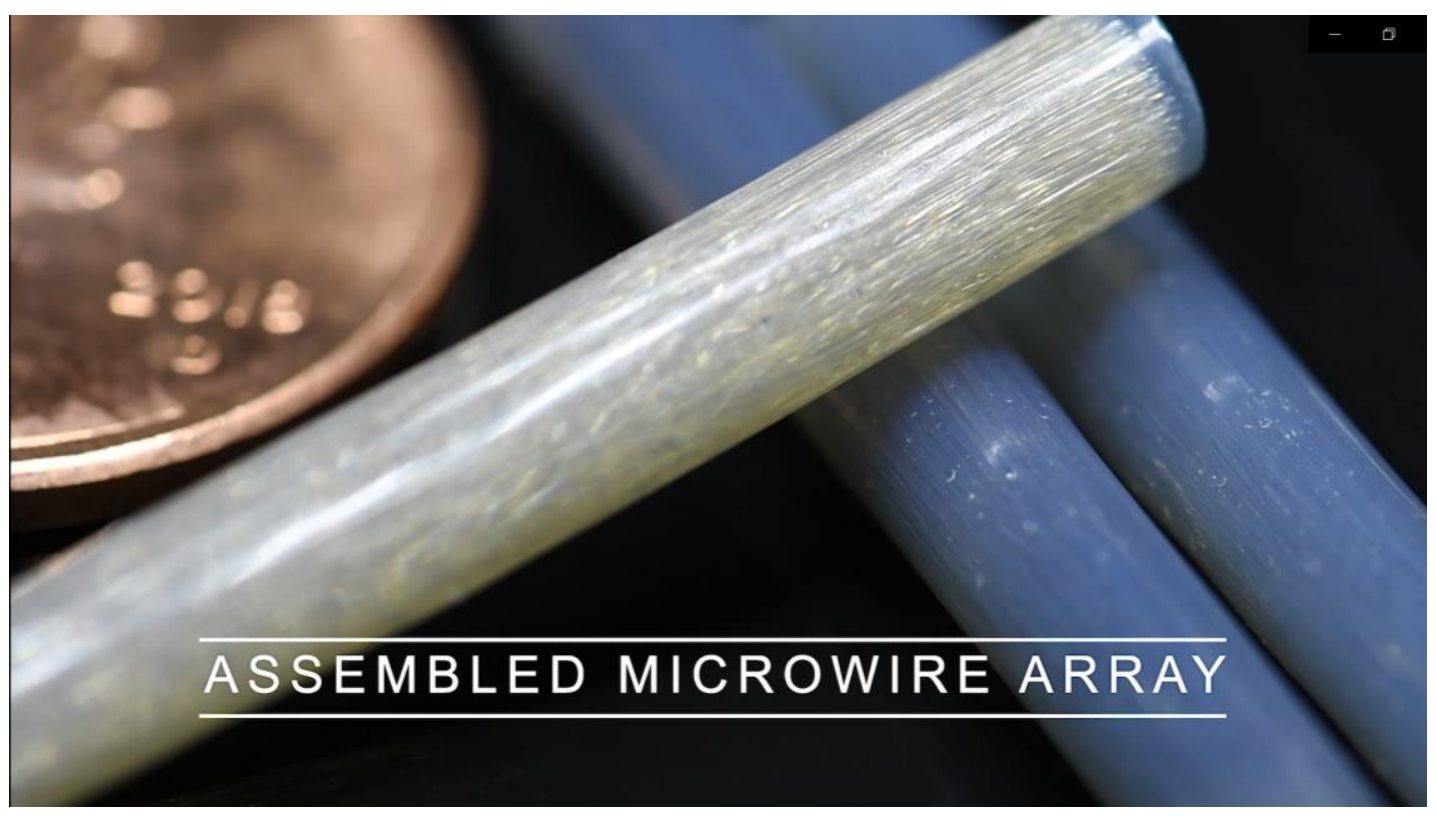

Figure 7. Assembled microwire arrays after electrodes were coated with spatial Parylene$\mathrm{C}$ coating and assembled in a clear heat shrink.

The array was then chemically etched in a piranha solution consisting of a 2:1 solution of $96 \%$ sulfuric acid and $30 \%$ hydrogen peroxide at $100^{\circ} \mathrm{C}$ and then oxygen plasma etched to reveal the bare wire. In order to keep the lengths of the exposed wire consistent across the entire array, wet and dry etches were alternated and repeated until $>1 \mathrm{~mm}$ of wire was exposed on the distal end of the bundle and 300-500 $\mu \mathrm{m}$ of wire was exposed on the proximal end. The electrodes were coated with a $1.5 \mu \mathrm{m}$ layer of Parylene-C insulation paired with silane A-174 for improved adhesion. Fluorescent epoxy created as a mixture of EPO-TEK 301 and fluorescent dye Y3G was used to embed the insulated wires in preparation for confocal microscopy during the later tip deinsulation step. The proximal end of the array was embedded in non-fluorescent EPOTEK 301. After curing at $65^{\circ} \mathrm{C}$ for 2 hours, both ends of the bundle of electrodes were machined down with a lathe until the wires were exposed on the proximal end of the 
bundle and until approximately $300 \mu \mathrm{m}$ of epoxy remained between the tips of the electrodes and the surface of the epoxy. To eliminate any tooling marks from machining, both ends of the bundle were lightly polished by hand on silicon carbide paper up to 1200 grit until the epoxy was clear enough to be seen beneath the surface as shown in Figure 8.

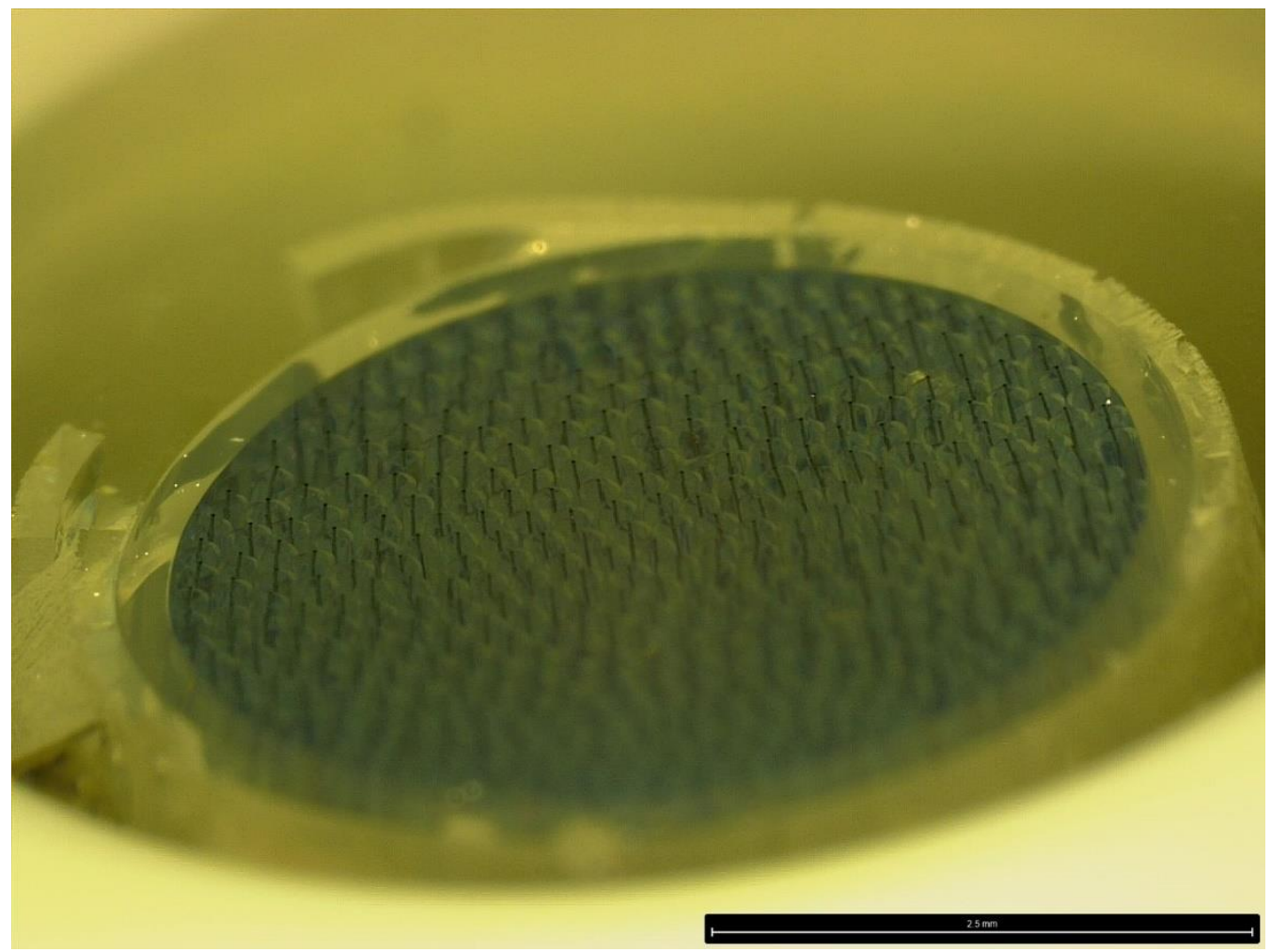

Figure 8. Proximal end of a $12 \mathrm{~mm}$ diameter, $200 \mu \mathrm{m}$ pitch bundle polished and with a small length of exposed wire prior for pressing onto a CMOS chip.

To expose recording sites on the microwires, laser ablation was utilized to deinsulate the tips of the electrodes. Confocal microscopy verified the distance, approximately 300 $\mu \mathrm{m}$, between the surface of the epoxy and the tips of the electrodes. Using this information, UV laser ablation was used to etch the epoxy to expose $10-30 \mu \mathrm{m}$ of the distal tips, based on calculations for optimal impedance for neural recordings. The remaining Parylene-C insulation covering the tips was etched away with oxygen plasma. 
The wires on the proximal end of the bundle were also etched with oxygen plasma to expose $\sim 10 \mu \mathrm{m}$ of bare wire for better connection with the CMOS chip. The electrodes were released from the epoxy with a final piranha etch until $800 \mu \mathrm{m}-1.5 \mathrm{~mm}$ of wire length was exposed as shown in Figure 9.

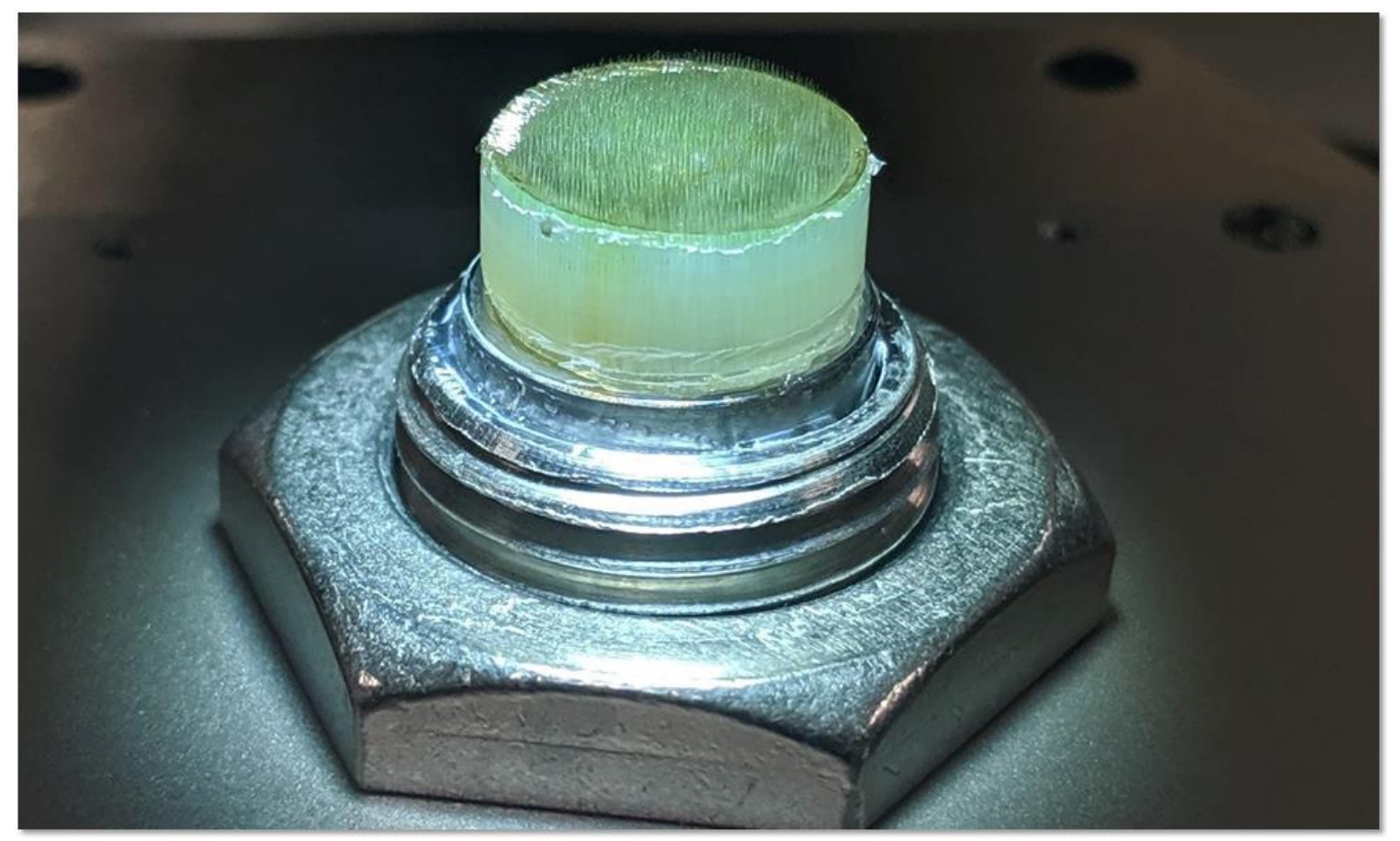

Figure 9. A fully fabricated microwire electrode array threaded onto the front plate ${ }^{(32)}$.

The arrays were imaged with a scanning electron microscope after the arrays were released from epoxy with piranha. Each array was examined to verify relatively uniform tip deinsulation, tip sharpness after processing, and the quality of the insulating layer. Photographs of these sharp tips were imaged with a scanning electron microscope, as shown in Figure 10. 

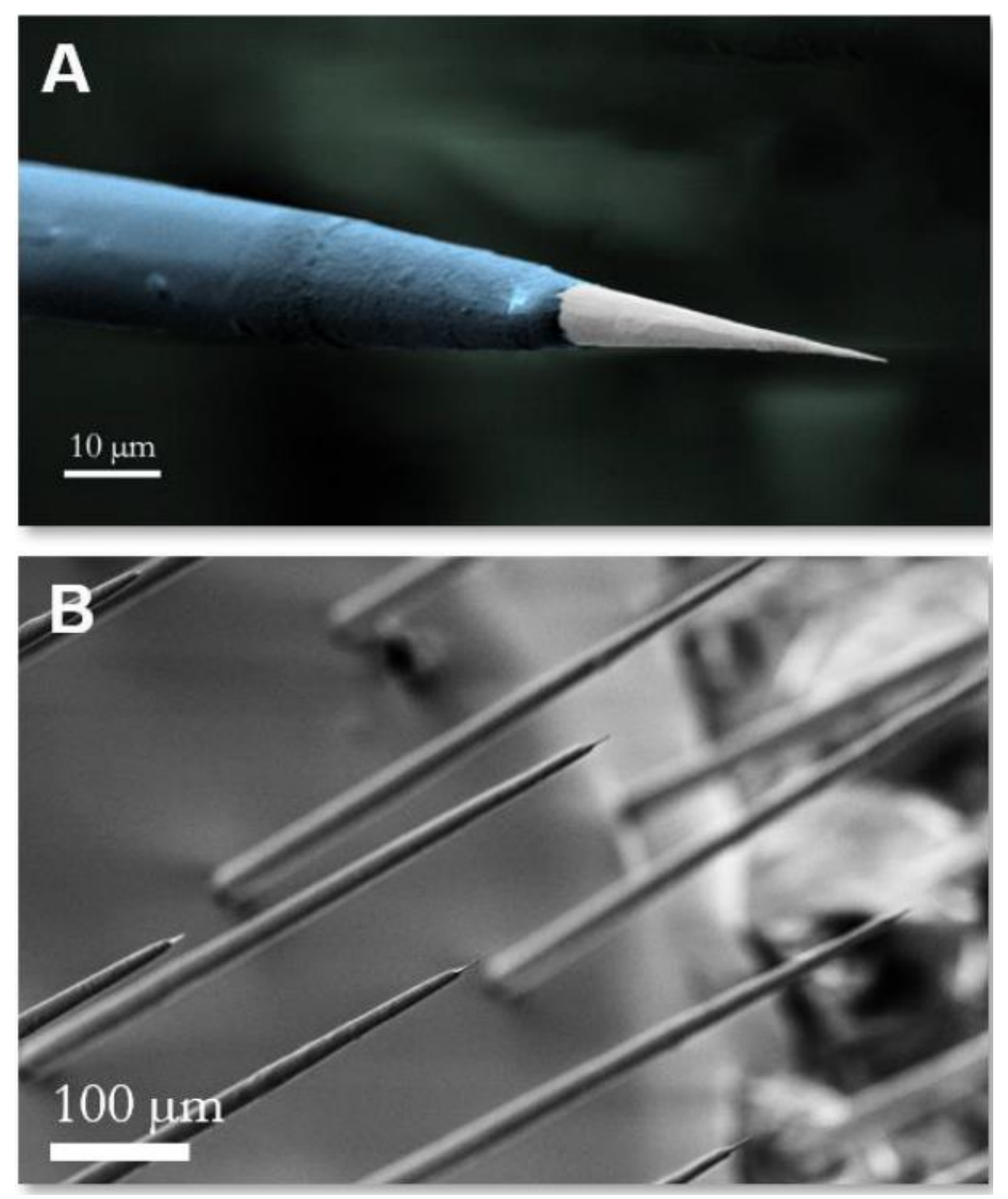

Figure 10. (A) False color image of an electrochemically sharpened microwire taken with a scanning electron microscope (B) Image of insulated microwire array taken with a scanning electron microscope ${ }^{(1)}$.

\subsubsection{Connecting to a Sensor}

The arrays were bonded to a CMOS amplifier array connected to the Argo System as described in Sahasrabuddhe et al., 2021. In order to do so, the stainless steel tube surrounding the array was first bonded with cyanoacrylate into a custom hollowed 3/4"20 threaded rod to screw into the center of the front plate with a flexible diaphragm 
holding the array in place. The front plate was loaded into the top jaw of a modified vise standing on its side. The CMOS chip was seated in a stainless steel housing that was loaded into the bottom jaw of the vise. The vise came together to press the back of the array onto the CMOS sensor. When the array presses down onto the CMOS chip, the connected user interface displays a map of connected pixels on the chip to visualize the connectivity of the array to the CMOS sensor. After a satisfactory connectivity was achieved, when further pressing no longer yielded a higher connectivity on the user interface, the front plate was screwed on four corners into the housing for the CMOS chip to secure the bundle in its mechanically coupled state, as shown in Figure 11.

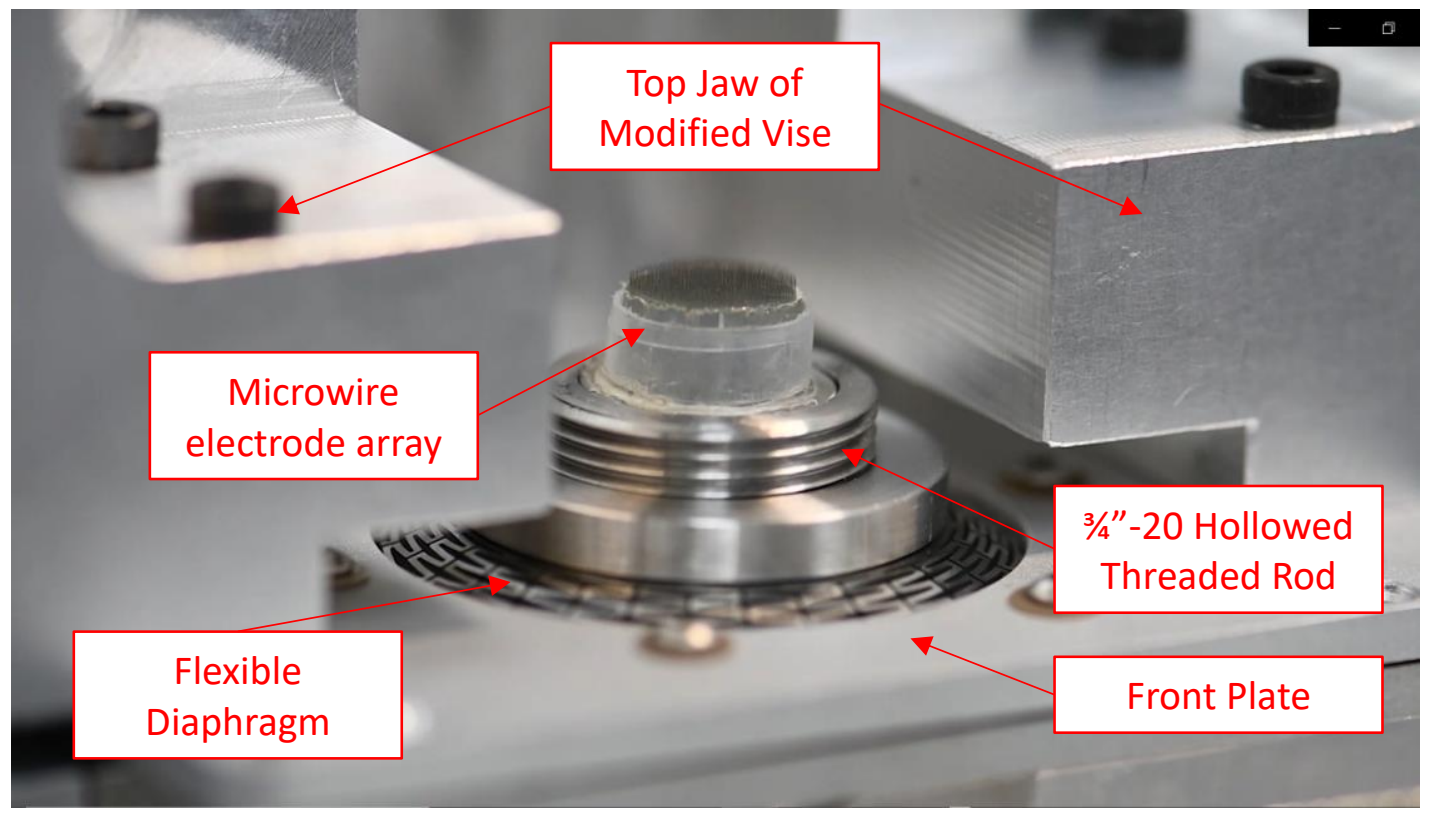

Figure 11. Microwire bundle array secured in vise during pressing onto CMOS sensor.

\subsubsection{Saline Bath Test}

In order to evaluate the connectivity, or percentage of microwires that successfully bonded to the CMOS sensor, the functionality of the array was tested for ohmic contact to the chip in a saline bath test. While connected to the Argo System, the tips of the 
electrode array were submerged into a phosphate-buffered saline (PBS) bath ${ }^{(10)}$. A 2 $\mathrm{kHz}, 2 \mathrm{mV}_{\mathrm{pp}}$ sine wave was applied through all the electrodes to verify connectivity to the CMOS chip, and a $2 \mathrm{kHz}, 0.5 \mathrm{mV}_{\mathrm{pp}}$ sine wave was applied to calculate pixel gain from the recorded voltage measurement ${ }^{(10)}$. Another voltage measurement was taken after shorting the saline bath to calculate the noise ${ }^{(10)}$. The recording was band-pass filtered between $300 \mathrm{~Hz}$ and $6 \mathrm{kHz}$ to isolate input-referred noise ${ }^{(10)}$. Channels with optimal gain and low noise were chosen to form a connectivity mask, like the one shown in Figure 12, to represent the electrodes in the array suitable for neural recording in vivo. This mask also served as a baseline measurement to compare with data acquired from neural recordings.
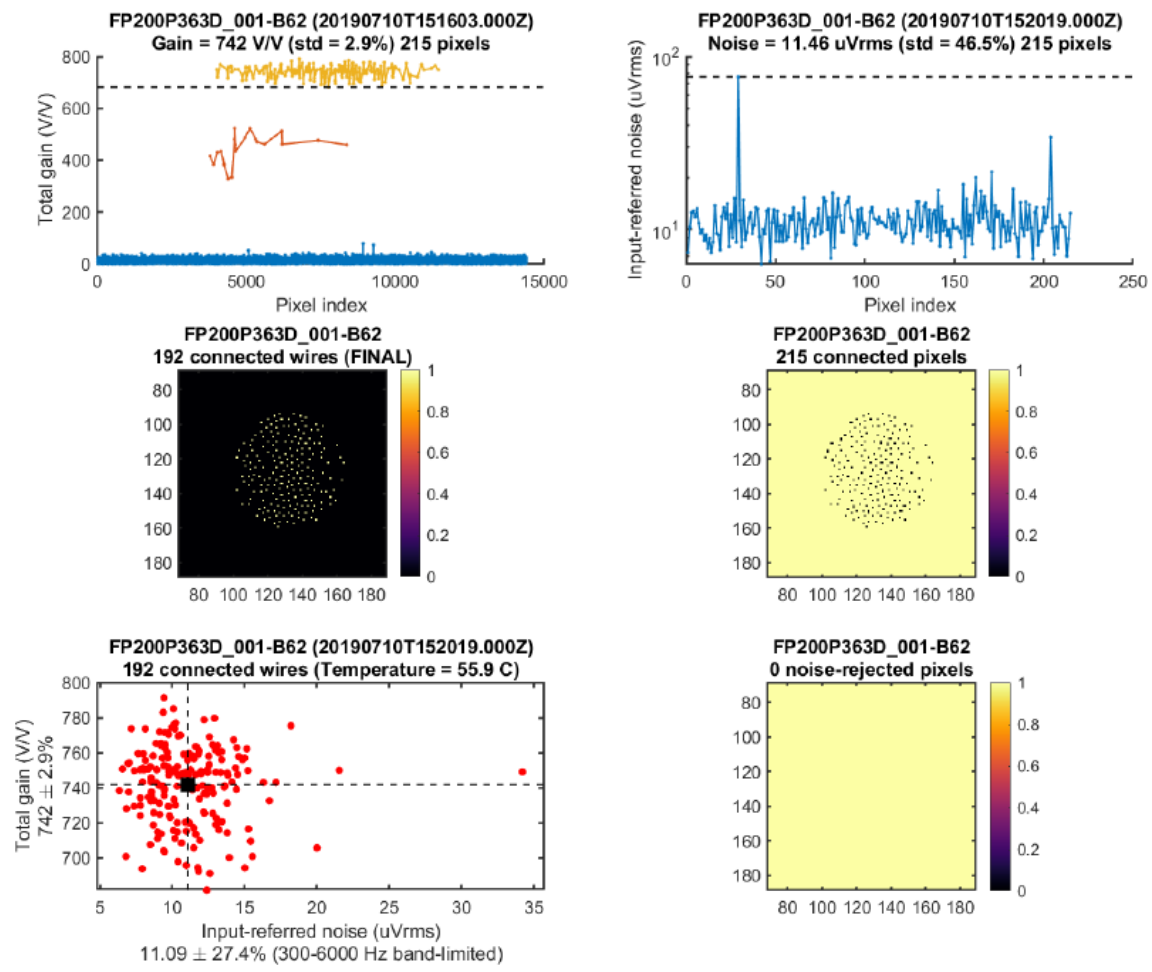

Figure 12. Example of a connectivity mask of a $3 \mathrm{~mm}$ diameter, $200 \mu \mathrm{m}$ pitch bundle showing final connected wires and pixels after filtering for gain and noise. 


\subsubsection{Calculating Percentage Connectivity}

The connectivity mask displays the number of connected electrodes and analyzes if they are well suited for recording neural signals. In order to find the percentage connectivity, the total number of wires per array must first be confirmed. Due to minor variations of $\pm 5 \%$ in the Parylene-C coating across the deposition chamber, the spatial coating may not always be precise, particularly in arrays with larger spacing and larger diameters. In conjunction, manual aggregation of the wires does not guarantee the perfect hexagonal form, meaning there are frequently differences between each bundle in terms of electrode pitch and number of wires per array. To confirm the number of wires within each array, images of the proximal end of the bundle were taken, processed to 8bit as shown in Figure 13, and analyzed via the particle counter function on ImageJ prior to pressing onto the CMOS chip. The percentage connectivity was calculated by dividing the number of wires connected, as indicated on the connectivity mask, by the total number of wires in each array.

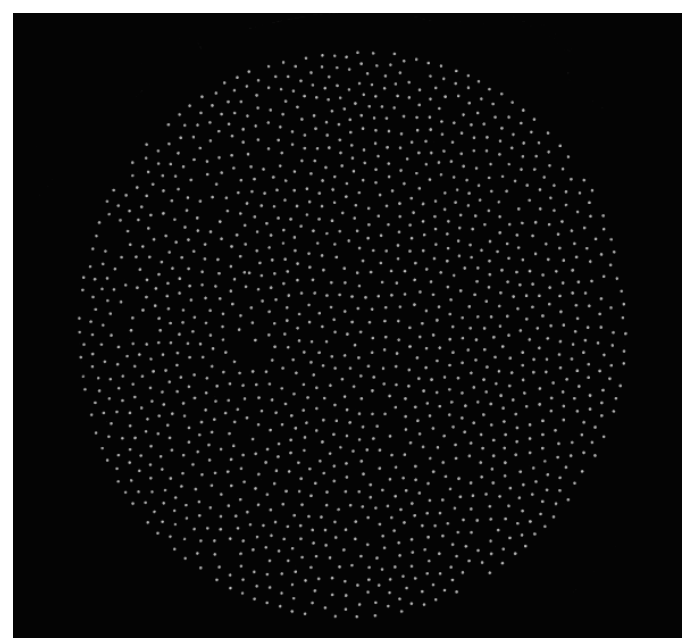

Figure 13. Example image of the proximal end of a $200 \mu \mathrm{m}$ array used to verify the number of wires within an array. 


\subsection{Safety}

Risks of fabricating electrodes using this process involved the use of some dangerous chemicals listed in Table 4. Most of the materials utilized in this process were relatively harmless. Phosphate buffered saline relatively safe, and splash goggles and gloves were worn while working with it. Calcium chloride, utilized in electrochemical processing, is a salt that minimally affects human health. While mixing the salt in water was not harmful, the electrochemical sharpening process caused the solution to emit a dangerous chlorine gas. In order to keep users safe, this process was done under a fume hood with appropriate filters to minimize exposure. Gloves and goggles were used regularly during this process as well.

Other chemicals that required additional care are the following. Silane A-174 is a skin sensitizer and care was taken to make sure there was no contact with skin. Gloves and goggles were used each time with Silane A-174. EPO-TEK 301 Part A and B and EPO-TEK H20E Part A and B are irritants, toxic, and corrosive in their uncured state, so gloves and safety goggles were worn each time when handling the epoxy as well. Common solvents, including acetone and isopropanol, are flammable and need to be kept away from an open flame. Gloves and goggles were worn while handling these chemicals, and they were stored in a fireproof cabinet. More hazardous chemicals, such as hydrogen peroxide and sulfuric acid, require additional precautions. While handling these chemicals in a specialized acid fume hood, protective face shields, primary nitrile gloves, secondary rubber gloves, tertiary vinyl gloves, and full-length aprons were worn to protect the user. Any vinyl gloves that came into contact with these chemicals were 
removed immediately and discarded into a specialized acid solid waste disposal, and secondary rubber gloves were thoroughly washed with water after each use to ensure the safety of future users. Aprons and face shields were examined prior to each use and replaced regularly to ensure the safety of users.

Upon contact with any of these materials, it is important to provide proper treatment in order to ensure little lasting damage to the health of the user. In case of eye contact, contact lenses must be removed and the eyes must be flushed with water for at least 15 minutes. If inhaled, the person utilizing the chemical must be moved to an area with fresh air and provided with artificial respiration if needed. When there is skin contact, it is recommended that the person flushes their skin with water and seek medical attention if their skin experiences any severe irritation.

Other hazards that are not related to chemicals include the utility of ovens, highenergy lasers, and machining equipment. Proper heat-resistant gloves were used while handling samples from ovens in order to ensure that users do not get burned. Laser cutter and other high-energy lasers raises the risk of vision damage if improperly used, so proper laser safety goggles were used each time. The laser was operated in a room with safety lights outside to indicate whether or not it was safe to enter the room. Heavy machining equipment, such as a lathe, are fast-spinning and dangerous. Users took extra care to ensure that there were no loose clothing items or hair that could potentially get caught on these tools. 


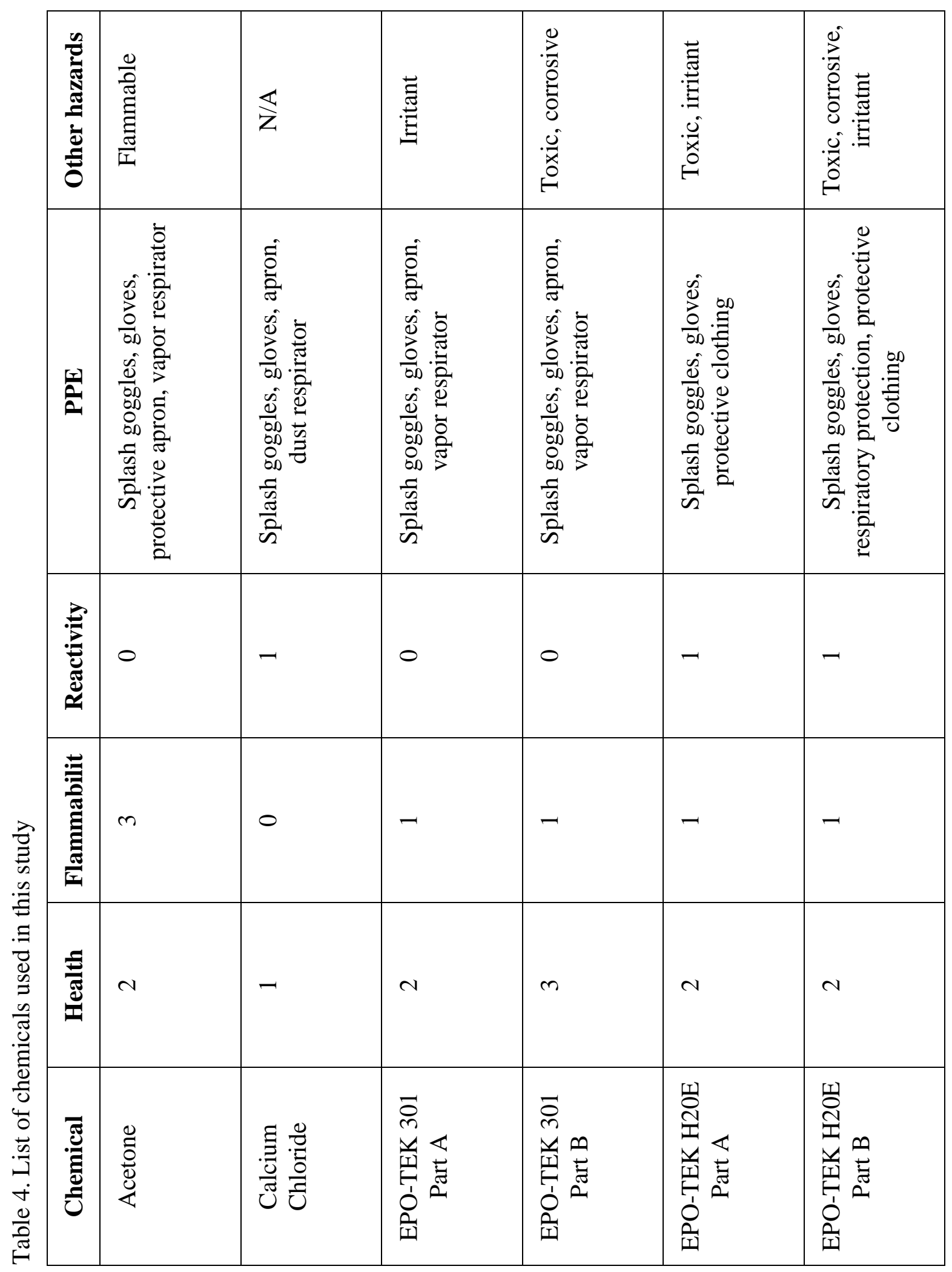




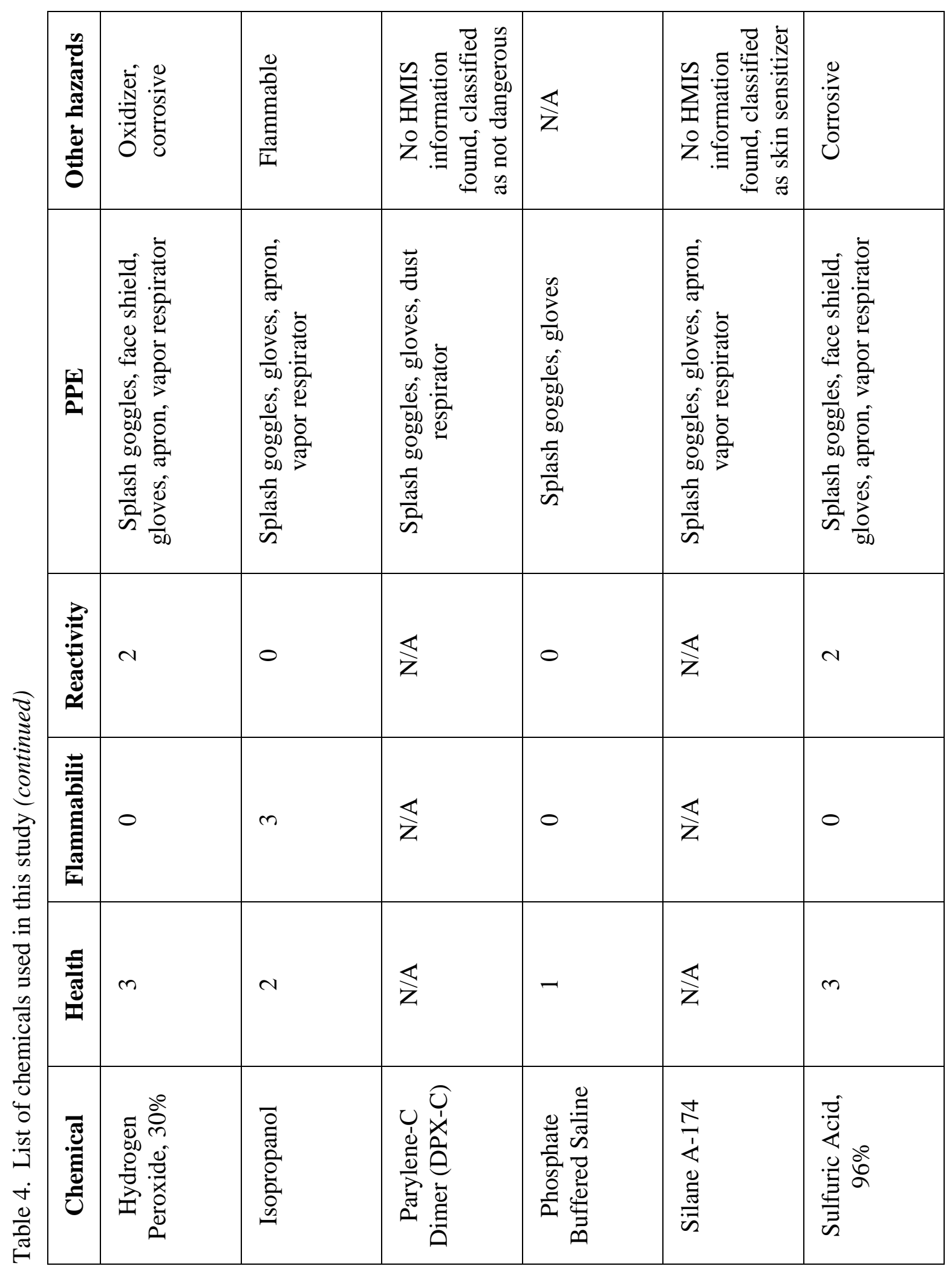




\section{RESULTS}

The percentage connectivity was measured for 21 different arrays. The raw data, including the total number of electrodes, number of connected electrodes, and the calculated percentage connectivity for each array, can be found in Appendix A. While all the electrodes demonstrated some percentage of connectivity, the mean connectivity of the entire sample $(n=21)$ was $71.64 \%$ with a standard deviation of 16.72 . The median connectivity of the sample was $68.98 \%$, seated between a minimum of $33.2 \%$ and a maximum of $97.92 \%$ connectivity. The arrays with lower conductivity tended to be ones that were built earlier in time, and the process was not yet as well developed, resulting in less ideal arrays with greater angular deviation on the proximal end. Because the mean and median were close, a normal distribution could be assumed. Graphing the percentage connectivity of every array built during this project on a normal probability plot in Figure 14 demonstrated that the data followed the theoretical normal distribution line relatively closely, meaning the sample approximately followed a normal distribution. The similarity between the mean and median further supported the assumption that the sample was normal. 


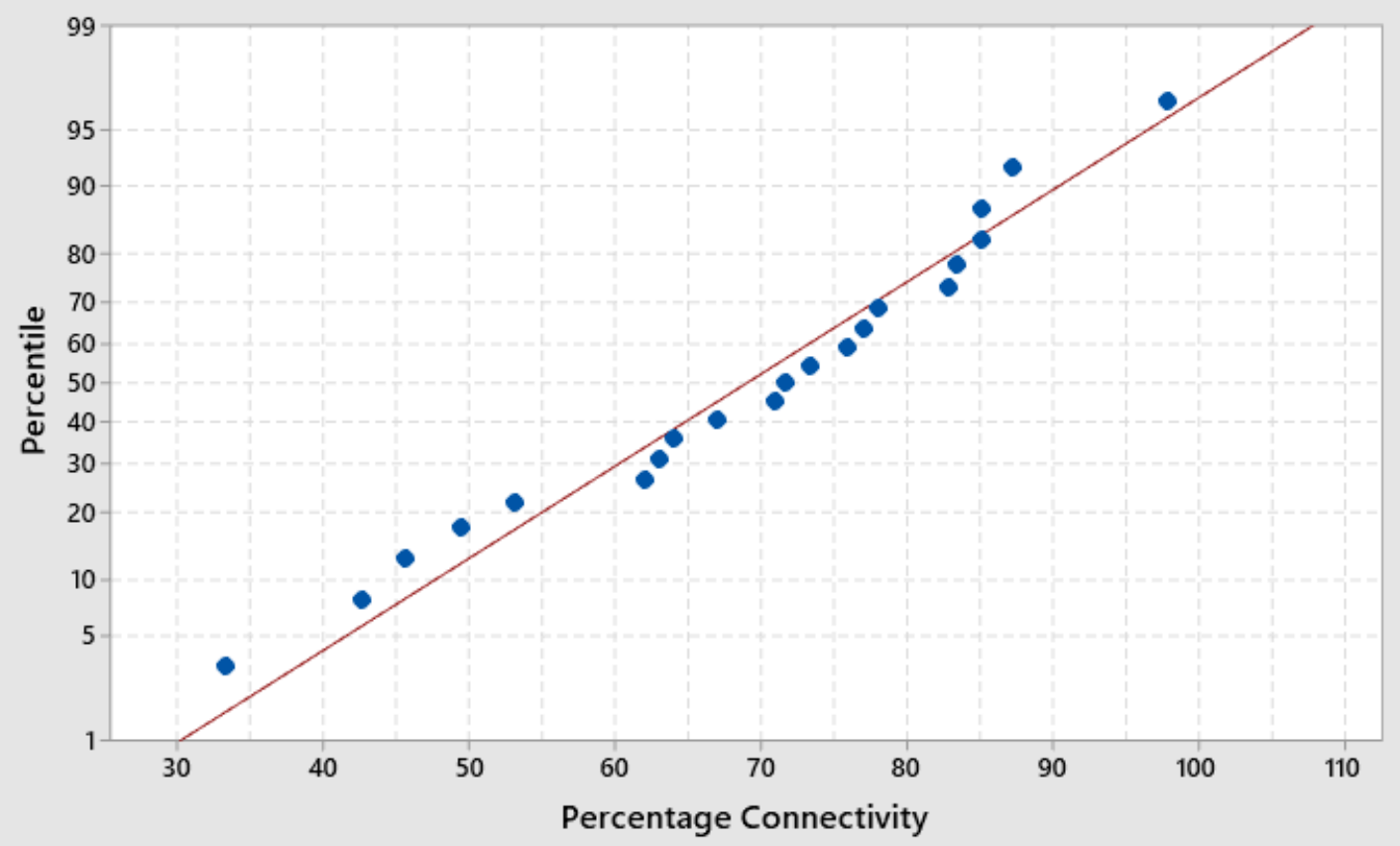

Figure 14. Normal probability plot of percentage connectivity of all microwire arrays.

\subsection{Array Diameter}

\subsubsection{Parametric Tests}

Bundles with diameters of $3 \mathrm{~mm}$ and $12 \mathrm{~mm}$ were bonded to evaluate the effect of increasing bundle size on connectivity. The $3 \mathrm{~mm}$ bundles $(\mathrm{n}=10)$ had an average connectivity of $63.08 \%$, represented by the white triangle outlined in black on the boxand-whiskers plot to the left in Figure 15, with a standard deviation of 16.46. The values ranged from a minimum of $33.20 \%$ to a maximum of $87.30 \%$. Meanwhile, the $12 \mathrm{~mm}$ bundles $(\mathrm{n}=11)$ had an average of $74.34 \%$, represented by a white triangle outlined in black on the box-and-whiskers plot to the right, connectivity with a standard deviation of 15.77. The values ranged from a minimum of $42.65 \%$ to a maximum of $97.92 \%$. Figure 15 demonstrates that the connectivity range of the two samples appeared similar. However, it appeared that the larger bundle, with a $12 \mathrm{~mm}$ diameter, had a greater mean 
and median percentage connectivity than the $3 \mathrm{~mm}$ diameter bundle. This was an unexpected result, since arrays with larger diameters were more prone to misalignment or angular deviations which may have caused fewer electrodes to connect. However, taking care to do all processing steps well minimized the possibility of defects that decreased connectivity. A $95 \%$ confidence interval for the mean connectivity was determined to be between $52.42 \%$ and $73.73 \%$ for $3 \mathrm{~mm}$ arrays and $62.18 \%$ and $84.50 \%$ for $12 \mathrm{~mm}$ diameter bundles.

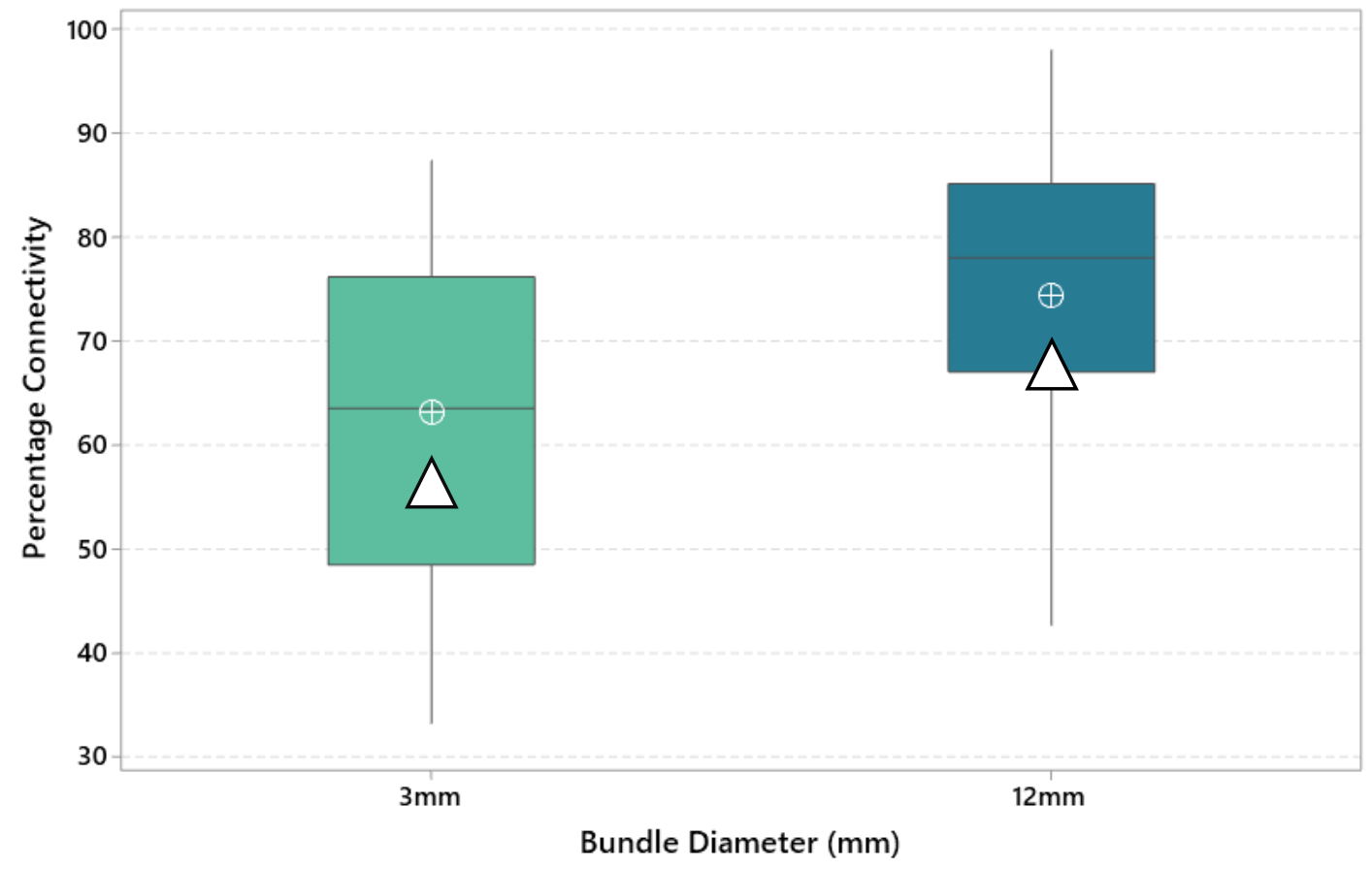

Figure 15. Box and whiskers plot showing the median, quartiles, and range of percentage connectivity of $3 \mathrm{~mm}$ and $12 \mathrm{~mm}$ diameter arrays.

To compare the two groups by testing the null hypothesis of similar average between two samples, one-way analysis of variance (ANOVA) with $\alpha=0.05$ was performed on the samples with MiniTab 19. The results reveal an F-value of $2.85(\mathrm{P}<0.126)$, as 
shown in Table 5, resulting in a failure to reject the null hypothesis; the means do not differ.

Table 5. Results of a one-way Analysis of Variance (ANOVA)

\begin{tabular}{|l|ccccc|}
\hline \multicolumn{1}{|c|}{ Source } & DF & Adj SS & Adj MS & F-Value & P-Value \\
\hline Factor & 1 & 665.7 & 664.7 & 2.56 & 0.126 \\
Error & 19 & 4924.2 & 259.2 & & \\
Total & 20 & 5588.9 & & & \\
\hline
\end{tabular}

Note: For $3 \mathrm{~mm}$ diameter and $12 \mathrm{~mm}$ diameter arrays

\subsubsection{Non-parametric Tests}

For these two samples, normality was assumed because of similar means and medians; however, in the event that the population is actually not normal, non-parametric tests were utilized to evaluate these samples. Percentile bootstrapping involves repeatedly sampling from the experimental data at random with replacement to construct surrogate datasets with the same sample sizes that allow for statistical inferences to be made ${ }^{(41)}$. Because the sample sizes were small and unequal, this method was particularly helpful. The connectivity data for $3 \mathrm{~mm}$ and $12 \mathrm{~mm}$ diameter bundles were randomly sampled with replacements to simulate a new data set from the pre-existing one, and the means of each new surrogate dataset was calculated. The difference between the new mean connectivity of the $3 \mathrm{~mm}$ and $12 \mathrm{~mm}$ samples were found and recorded. This process was completed 10,000 times in MATLAB to estimate the expected difference in each measurement and allowed for an even distribution of $3 \mathrm{~mm}$ and $12 \mathrm{~mm}$ diameter bundles and recorded in Figure 16. Based on these data, a 95\% confidence interval was calculated and shown to bound between the red lines indicated as 5\% CI. The null hypothesis, $\mathrm{H}_{0}$ mean and indicated in blue in Figure 16, of no difference in means 
between the two samples can be rejected if the observed difference fell outside the $2.5 \%$ to $97.5 \%$ percentile range of the bootstrapped distribution. However, as shown in Figure 16 , this was not the case, since the null hypothesis fell within the $95 \%$ confidence interval indicating that there is no statistical difference at $\alpha=0.05$ between the two means.

Hence, the hypothesis that the two samples have the same population mean cannot be rejected, even using non-parametric statistical analysis.

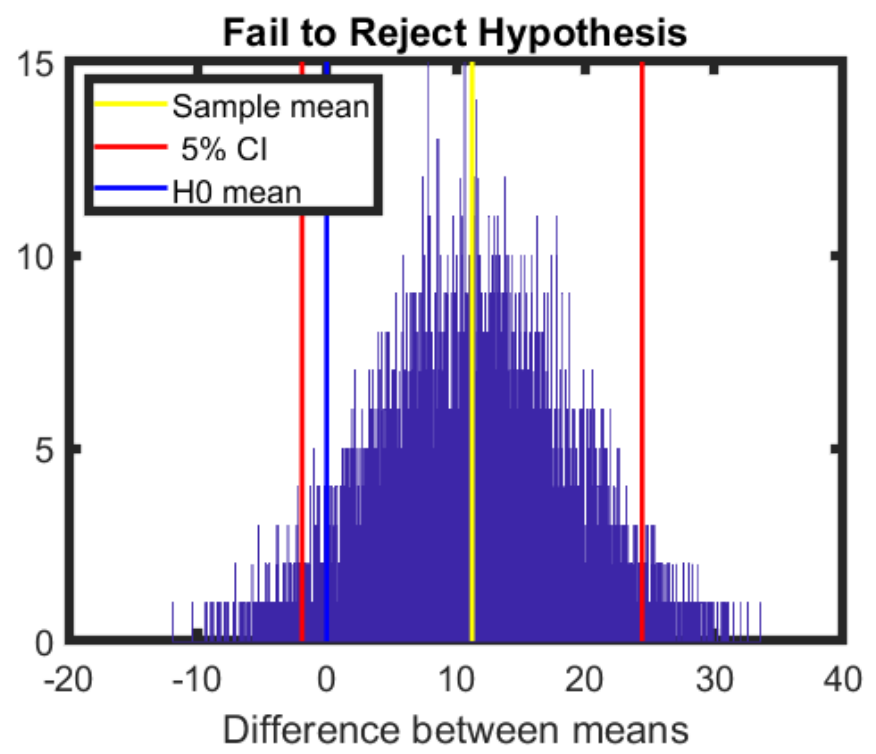

Figure 16. Percentile bootstrapping of the difference between means of $3 \mathrm{~mm}$ and $12 \mathrm{~mm}$ diameter bundles.

\subsection{Electrode Density}

\subsubsection{Parametric Tests}

The effect of electrode density on the connectivity of a microwire array was evaluated by comparing how well $12 \mathrm{~mm}$ diameter bundles with $200 \mu \mathrm{m}$ and $100 \mu \mathrm{m}$ wire-to-wire pitch connected to the CMOS chip. The $200 \mu$ m pitch bundles $(n=6)$, with connectivity values ranging from $70.92 \%$ to $85.12 \%$, demonstrated an average of $78.65 \%$ connectivity and a standard deviation of $6.19 \%$. The $100 \mu \mathrm{m}$ pitch bundles $(\mathrm{n}=5)$ had a $64.92 \%$ 
connectivity on average, ranging from a minimum of $42.65 \%$ to a maximum of $97.92 \%$, with a standard deviation of $22.64 \%$. Figure 16 shows a vast difference in range between the two samples; while the sample of $200 \mu \mathrm{m}$ pitch arrays have a range of $14.2 \%$, the difference between the greatest and least connected of $100 \mu \mathrm{m}$ pitch arrays was $55.27 \%$. This large difference in variation was also reflected in the difference between the standard deviations of the two samples. The means of the two samples were also different at $\alpha=0.05$. Figure 17 indicates that the $200 \mu \mathrm{m}$ pitch arrays had a higher average connectivity than the $100 \mu \mathrm{m}$ pitch arrays. The $95 \%$ confidence interval of the mean connectivity was also calculated to be between $64.08 \%$ and $93.23 \%$ for the $200 \mu \mathrm{m}$ pitch arrays and between $52.3 \%$ and $85.1 \%$ for the $100 \mu \mathrm{m}$ pitch bundles.

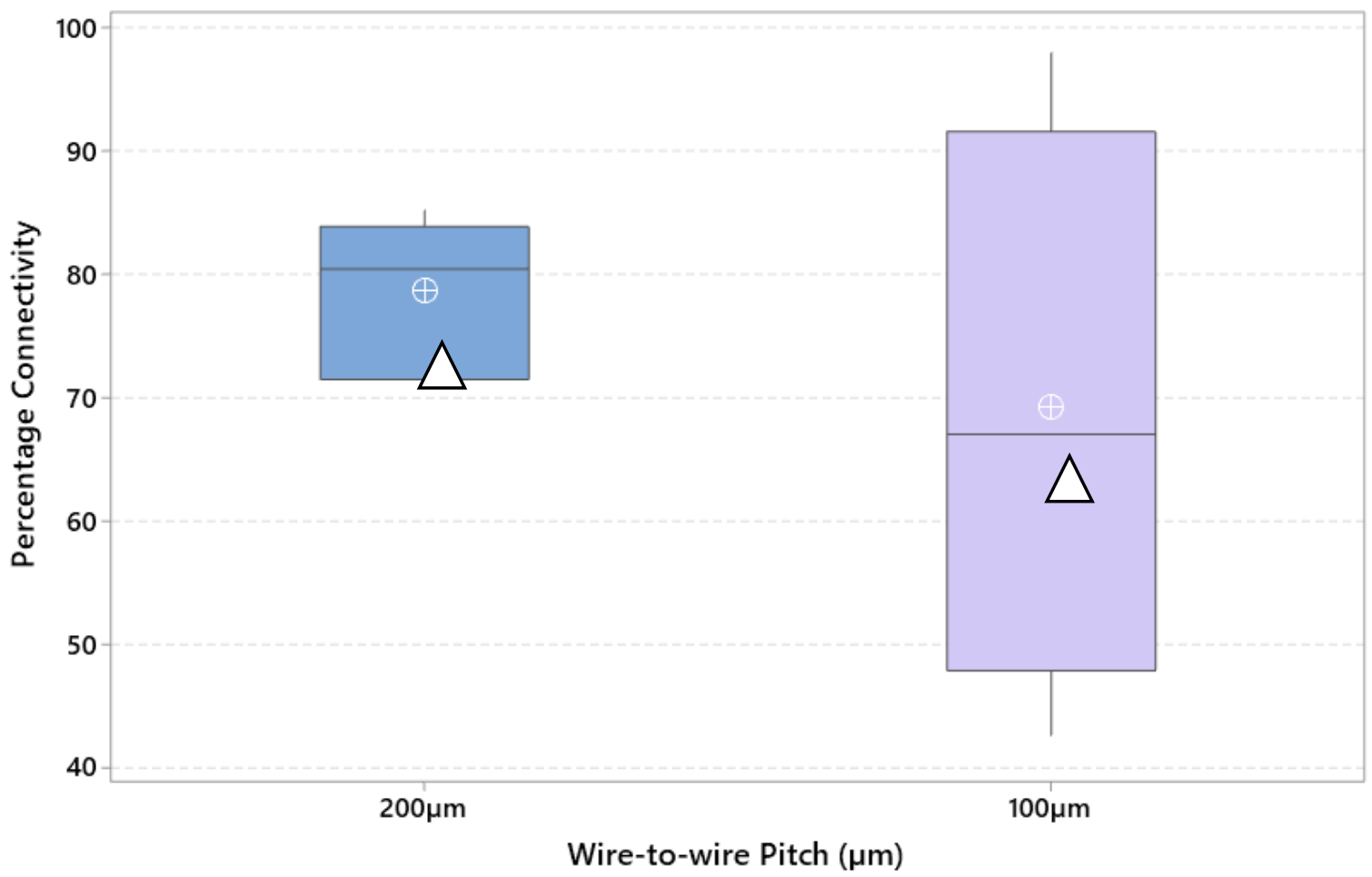

Figure 17. Box and whiskers plot showing the median, quartiles, and range of percentage connectivity of $200 \mu \mathrm{m}$ and $100 \mu \mathrm{m}$ pitch arrays. 
Similarly, ANOVA with $\alpha=0.05$ was also performed on these samples and are displayed in Table 6. The results show a F-value of 0.99 ( $\mathrm{P}<0.347)$, which is smaller than the F-critical value of 6.26. This indicates a failure to reject the null hypothesis of dissimilar population means. However, the small sample sizes render it difficult to draw more reliable conclusions.

Table 6. Results of a one-way Analysis of Variance (ANOVA)

\begin{tabular}{|c|ccccc|}
\hline Source & DF & Adj SS & Adj MS & F-Value & P-Value \\
\hline Factor & 1 & 245.4 & 245.4 & 0.99 & 0.347 \\
Error & 9 & 2241.3 & 249.0 & & \\
Total & 10 & 2486.7 & & & \\
\hline
\end{tabular}

Note: At $\alpha=0.05$ for $200 \mu \mathrm{m}$ and $100 \mu \mathrm{m}$ pitch arrays

\subsubsection{Non-parametric Tests}

Because the samples evaluating the effects of density on connectivity were also very small, non-parametric tests were similarly used for analysis. Like the sample sizes used to evaluate diameter, the sample sizes for density were small and unequal, and percentile bootstrapping simulated repeated experiments 10,000 times in MATLAB to estimate the expected difference in each measurement and compensated for differences in sample size between $200 \mu \mathrm{m}$ and $100 \mu \mathrm{m}$ pitch arrays. The observed difference did not fall outside the $95 \%$ confidence interval, as indicated by Figure 18, failing to reject the null hypothesis that the two samples do not have different means. 


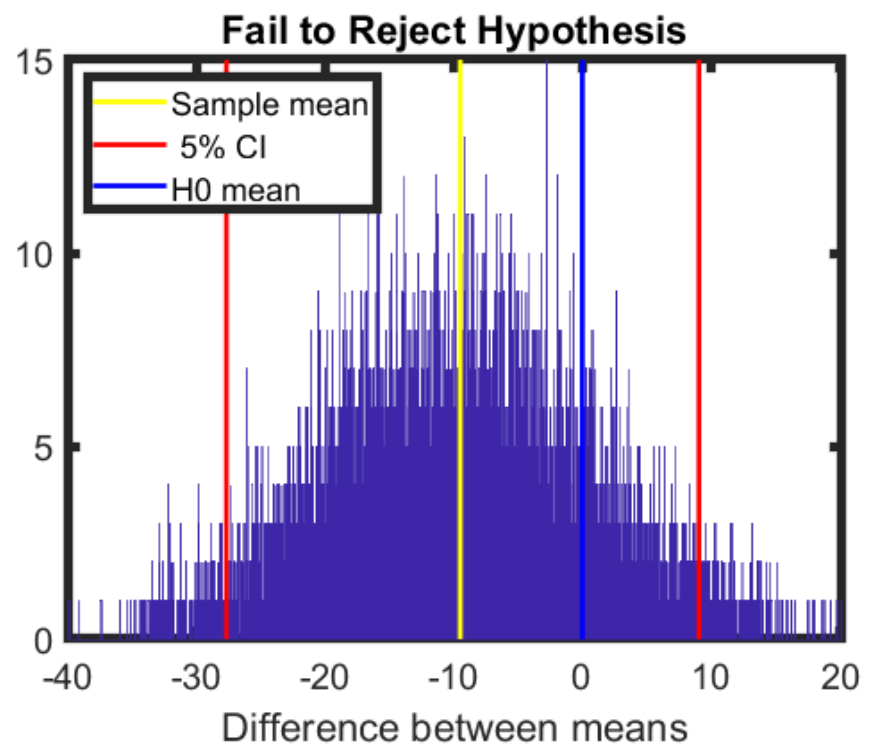

Figure 18. Percentile bootstrapping of the difference between means of $200 \mu \mathrm{m}$ and 100 $\mu \mathrm{m}$ pitch arrays. 


\section{DISCUSSION}

\subsection{Array Diameter}

The scalability of arrays by increasing bundle diameter was evaluated by analyzing the connectivity of $3 \mathrm{~mm}$ and $12 \mathrm{~mm}$ diameter bundles to a CMOS sensor. While the average connectivity and range of responses for $12 \mathrm{~mm}$ bundles appear slightly greater than the average connectivity and responses for $3 \mathrm{~mm}$ arrays, ANOVA indicated that there was no statistical difference between the means of the two samples. Similarly, the observed difference in the bootstrapped test did not fall outside the confidence interval either. Both parametric and non-parametric tests failed to reject the null hypothesis that the two populations have the same mean percentage connectivity, indicating that there was no statistically significant difference between the average connectivity of the $3 \mathrm{~mm}$ diameter and $12 \mathrm{~mm}$ diameter arrays.

Increasing the array diameter does not come without its issues. A minor angular deviation on the proximal end of the array that otherwise would not be apparent in a 3 $\mathrm{mm}$ array with a smaller radius would be magnified in a $12 \mathrm{~mm}$ array with a larger radius due to amplification of angular deviation across a larger surface area. For example, a one degree deviation across a $3 \mathrm{~mm}$ diameter array from end to end would result in a $52 \mu \mathrm{m}$ height difference across the surface, while the same angular deviation across a $12 \mathrm{~mm}$ diameter array from end to end would result in a $209 \mu \mathrm{m}$ height difference. Any nonplanarity negatively affects connectivity, as the electrodes would not properly interface with the CMOS chip if some parts of the bundle do not make contact with the pixels. Often, this is visible in larger arrays when a crescent moon-shaped shadow of no 
connection appears along the outer perimeter of the connectivity map due to a bevel on the bundle. Any deviation and misalignment of the array on the CMOS sensor would also result in poor connectivity, particularly in larger electrode bundles with diameters close to the dimensions of the chip. With smaller diameter arrays, misalignment would likely play a far smaller role in connectivity issues, because the surface area of the proximal end of the array is much smaller than the surface area of the CMOS array. Minor deviations of even several hundred microns, in centering the array on the CMOS sensor would not be detrimental to the connectivity, since the surface area of the CMOS array was larger and could compensate for any slop. Bundles with diameters close to the maximum dimensions of the CMOS sensor must be aligned carefully such that the electrode array was perfectly centered on the chip; otherwise microwires on the periphery of the bundle may not make contact with the pixels on the sensor.

Another factor that may play a role in connectivity is the pressing force of the array onto the CMOS chip. Larger arrays have a greater number of wires and surface area to contact with the pixels, requiring a greater amount of force to fully connect the entire bundle. Optimal connectivity was achieved by securing the bundle onto the CMOS chip when it appears that most, if not all, of the array is within contact with the pixels. This technique allows for force required to connect each array to be tailored to that bundle and varies from array to array, causing differences in percentage connectivity.

\subsection{Electrode Density}

Microwire electrode array scalability was also examined by varying electrode density. Decreasing the wire-to-wire distance increased the array density. The $200 \mu \mathrm{m}$ pitch 
bundles had a higher average percentage connectivity than $100 \mu \mathrm{m}$ pitch arrays. The 100 $\mu \mathrm{m}$ pitch arrays have a substantially higher variance and range of responses than the less dense bundles. This indicates that while $100 \mu \mathrm{m}$ pitch arrays demonstrate high percentage connectivity, the $200 \mu \mathrm{m}$ pitch bundles are more consistent. However, the failure to reject the null hypothesis that the two populations have the same mean percentage connectivity, despite these differences, demonstrating that decreasing from $200 \mu \mathrm{m}$ and $100 \mu \mathrm{m}$ pitch show no statistically significant change in average connectivity.

Electrode density also affects the pressing force required to connect the array to the CMOS. More wires require a greater amount of force to connect the array onto the pixels, as previously discussed regarding increasing array size. Bonding force is affected by electrode density as well, as decreasing the wire-to-wire distance increases the number of wires within a bundle. As mentioned before, the custom interfacing allows the amount of force required to be individual to each bundle, causing differences in percentage connectivity depending on how much force is required.

\subsection{Other Limitations}

Other possible variables that were not considered within this study include the human factors. Each of these bundles were assembled by hand, resulting in the possibility of differences between each array. As more arrays were made, the expertise in handling thin microwires and delicate electronics improved, simultaneously improving the quality of the bundles and the connectivity. It would be difficult to quantify and evaluate how strong of a role human factors played into creating arrays with greater 
connectivity, including how chronology of assembly of the arrays in these samples affected the percentage connectivity may be interesting to examine in another study. 


\section{RECOMMENDATIONS FOR FUTURE RESEARCH}

Due to the limitations of this project, future research is recommended for more conclusive results. To obtain statistically significant results, the experimental set up needs to be well-defined and more intentional. This chapter outlines recommendations for a more robust experimental design and analysis that would yield more meaningful conclusions. The recommended set up would be important for optimizing the process for mass-producing commercial electrode arrays by understanding the limitations of the manufacturing process.

\subsection{Design of Experiments}

\subsubsection{Research Question and Hypothesis}

The results of this project failed to disprove the null hypothesis, forcing its acceptance. This did not allow strong, meaningful conclusions to be drawn. Should the size of the effect be too small to statistically discern, increasing sample sizes in further studies or decreasing the significance level of the statistical analysis will yield more conclusive results.

A strong design of experiments first requires a clearly defined research question, null, and alternative hypotheses. The goal of this project is to analyze if array diameter and array density affect the percentage connectivity of the array when pressed on a CMOS chip. If so, how much do the factors and their interaction affect the response? In this case, the response variable in question is the percentage connectivity, while the factors are the array diameter and array density. The null hypothesis is that the array diameter and density, along with their interaction (array diameter*array density), will not affect the 
percentage connectivity. The alternate hypothesis will be that the array diameter and density, along with their interaction (array diameter*array density) will affect the percentage connectivity when pressed onto a CMOS chip. While the hypothesis is similar to that of the previous study, the results may be strengthened by increasing the sample size to produce results of a higher power, making inconclusive assessments less likely.

\subsubsection{Experimental Design}

The two factors in question include array diameter (A) and array density (B), and each factor needs to be evaluated at low and high levels. Microwire electrode array diameters should be $3 \mathrm{~mm}$ and $12 \mathrm{~mm}$. Arrays should also be made in low density (200 $\mu \mathrm{m}$ pitch) and high density (100 $\mu \mathrm{m}$ pitch). This information is summarized in Table 7. Table 7. Summary of Factors and Their Levels

\begin{tabular}{|c|c|c|c|}
\hline Factor & $\begin{array}{c}\text { Letter } \\
\text { Corresponding to } \\
\text { Factor }\end{array}$ & Low Level & High Level \\
\hline Diameter & A & $3 \mathrm{~mm}$ & $12 \mathrm{~mm}$ \\
\hline Density & B & $200 \mu \mathrm{m}$ Pitch & $100 \mu \mathrm{m}$ Pitch \\
\hline
\end{tabular}

A $2 \times 2$ factorial experiment should be used to test how the main effects and interaction affect response variable percentage connectivity. The main effects are diameter (A) and density (B). The two-way interaction between high levels of A*B should also be tested. Lastly, the low level of the two factors should be tested as well. Altogether, there should be 4 different groups of conditions to be evaluated within this experiment with 2 levels for each factor, or 1 degree of freedom for each factor and their interaction. Assuming a power of $80 \%$, statistical significance of $\alpha=0.05$, and an assumed medium effect size of 
0.25 , a calculated total sample size of 128 , as calculated with $\mathrm{G}^{*}$ Power, is needed to produce meaningful results ${ }^{(42,43)}$. This will mean each set of conditions would require 32 iterations. In an optimal project, this sample size would generate strong results, but such a costly project would likely not be feasible from a business perspective unless these arrays were intended to enter production for commercial purposes. The arrays in this project were primarily built as a part of a proof-of-concept for a human implantable device and were not meant to be mass produced, but a similar study may be done to optimize specifications for a future commercial device.

To eliminate chronology from affecting the response, the run order of the experiments must be randomized. To create a completely randomized order of which these arrays must be fabricated and evaluated, the number of factors and replicates should be programmed into MiniTab to build a randomized final design, including the order of which the responses should be recorded. Blocking can be done to ensure that extraneous factors do not affect the response variable, but because each array is fabricated independently, it is unnecessary to build blocks and should be run as a full factorial design.

\subsection{Statistical Analysis}

\subsubsection{Two-way Analysis of Variance}

Results should be analyzed in Minitab to obtain a table of coded coefficients, where preliminary significance and effectiveness can be analyzed. True significance should be later evaluated via two-way ANOVA and the normal probability table. Minitab 
calculates the effects of each factor and interaction and provides graphs to help visualize the effects between low and high levels of each factor.

While these graphs help describe how different factors may alter the response, it is also critical to evaluate the significance of these factors and interactions to understand if they truly matter. Two-way ANOVA should be done in Minitab. P-values, or probability values, are calculated by obtaining the sum of squares. Mean squares is calculated through the sum of squares. F-values should be calculated through mean square values. The P-value determines whether these factors or interactions will play a role in affecting the percentage connectivity at a specific significance level. In this case, $\alpha=0.05$, meaning a P-value below 0.05 will be deemed significant, and would be sufficient for drawing strong statistical conclusions.

\subsubsection{Regression Model}

A preliminary regression model, taking the coefficients calculated in the effects table before, can be built. An intercept can be calculated as a mean of all the observations. The data can then be evaluated against this model for an $\mathrm{R}^{2}$ value, an indicator of how much variability exists within the sample. Ideally, a model that most accurately represents a sample has an $\mathrm{R}^{2}$ value close to $100 \%$.

\subsubsection{Error Analysis}

The observations must be evaluated for any outliers within the sample as well as any trends due to experimental chronology. A normal probability plot of all the observations should be plotted against their residuals to observe any major outliers. If not, normality in the errors can also be assumed for the samples. Alternatively, the data can be 
evaluated through a histogram to visualize whether the sample follows roughly a normal distribution. This will also make outliers visible within the distribution. To evaluate whether chronology plays a role in the response, the residuals of the responses can be plotted in order of observation. Ideally, no visible patterns should be observed within this test, demonstrating that timing and experimental order did not affect the percentage connectivity.

\subsection{Summary}

Further studies may choose to focus on process optimization to produce the most value from these costly studies from a manufacturing standpoint. Understanding whether factors, such as array and diameter, affect percentage connectivity is important, but it would be more valuable to understand how these factors affect connectivity and whether the interactions do as well. Two-way Analysis of Variance enables this by comparing the results of low and high levels of each factor to show possible trends and interactions between these factors. Further improvements for these studies involve intentional randomization of experimental order to reduce biases and see if chronological trends in results exist. Should the results of future studies be inconclusive, the sample sizes may need adjustment to provide a study with more statistical power. The pre-assigned low and high levels of the factors may also need reevaluation, since the effects may be too small to yield meaningful results. These studies are critical, particularly when scaling up for producing mass-manufactured, commercialized medical devices for understanding process limitations and capabilities. 


\section{CONCLUSION}

Intracortical neuron recordings are invaluable due to their high temporal resolution. Microwire electrode arrays, when paired with a CMOS amplifier array such as within the Argo System, are a scalable, cost-efficient modality for detecting neural signals.

Microwire arrays can be scaled up by increasing the diameter and decreasing the pitch between the wires, because there is no significant difference in extent of connectivity between bundles of different sizes and densities. By quantifying this approach toward creating intracortical neuron recordings, new arrays with greater recording capabilities can be produced. Future experiments exploring novel materials and new fabrication schemes for bundled arrays can improve upon the current process. Improvements to build high-bandwidth neural interfaces open new doors to allow patients with significant mobility disorders and sensory impairment to regain their independence by communicating directly with a computer and the outside world. 


\section{REFERENCES}

1. Müller-Putz, G.R., and G. Pfurtscheller. Control of an Electrical Prosthesis With an SSVEP-Based BCI. IEEE Transactions on Biomedical Engineering. 55(1):361364, 2008.

2. Cincotti, F., D. Mattia, F. Aloise, S. Bufalari, G. Schalk, G. Oriolo, A. Cherubini, M.G. Marciani, and F. Babiloni. Non-invasive brain-computer interface system: Toward its application as assistive technology. Brain Research Bulletin. 75:796-803, 2008.

3. Sellers, E.W., T.M. Vaughan, and J.R. Wolpaw. A brain-computer interface for long-term independent home use. Amyotrophic Lateral Schlerosis. 11:449-455, 2010 .

4. Nicolas-Alonso, L.F., and J. Gomez-Gil. Brain Computer Interfaces, a Review," Sensors. 12:1211-1279. 2012.

5. Ball, T., M. Kern, I. Mutschler, A. Aertsen, and A. Schulze-Bonhage. Signal quality of simultaneously recorded invasive and non-invasive EEG. NeuroImage, 46:708-716, 2009.

6. Schalk, G., J. Kubanek, K.J. Miller, N.R. Anderson, E.C. Leuthardt, J.G. Ojemann, D. Limbrick, D. Moran, L.A. Gerhardt, and J.R. Wolpaw. Decoding two-dimensional movement trajectories using electrocorticographic signals in humans. J. Neural Eng. 4:264-275, 2007.

7. Kubie, J.L. A Driveable Bundle of Microwires for Collecting Single-Unit Data from Freely-Moving Rats. Physiology \& Behavior. 32:115-118, 1984.

8. Nicolelis, M.A.L., D. Dimitrov, J.M. Carmena, R. Crist, G. Lehew, J.D. Kralik, and S.P. Wise. Chronic, multisite, multielectrode recordings in macaque monkeys. Proc. Natl. Acad. Sci. USA. 100(19):11041-11046, 2003.

9. Nolta, N.F., M.B. Christensen, P.D. Crane, J.L. Skousen, and P.A. Tresco. BBB leakage, astrogliosis, and tissue loss correlate with silicon microelectrode array recording performance. Biomaterials. 53:753-762, 2015.

10. Sahasrabuddhe, K., A.A. Khan, A.P. Singh, T.M. Stern, Y. Ng, A. Tadi ć, P. Orel, C. LaReau, D. Pouzzner, K. Nishimura, K.M. Boergens, S. Shivakumar, M.S. Hopper, B. Kerr, M.E.S. Hanna, R.J. Edgington, I. McNamara, D. Fell, P. Gao, A. Babaie-Fishani, S. Veijanlainen, A.V. Klekachev, A.M. Stuckey, B. Luyssaert, T.D.Y. Kozai, C. Xie, V. Gilja, B. Dierickx, Y. Kong, M. Straka, H.S. Sohal, and 
M.R. Angle. The Argo: A high channel recording system for neural recording in vivo. J. Neural Eng. 2020.

11. Schwarz, D.A., M.A. Lebedev, T.L. Hanson, D.F. Dimitrov, G. Lehew, J. Meloy, S. Rajangam, V. Subramanian, P.J. Ifft, Z. Li, A. Ramakrishnan, A. Tate, K. Zhuang, and M.A.L. Nicolelis. Chronic, Wireless Recordings of Large Scale Brain Activity in Freely Moving Rhesus Monkeys. Nat. Methods. 11(6):670-676, 2014.

12. Tseng, W.T., C.T. Yen, and M.L. Tsai. A bundled microwire array for long-term chronic single-unit recording in deep brain regions of behaving rats. Journal of Neuroscience Methods. 201:368-376, 2011.

13. Misra, A., J.F. Burke, A. Ramayya, J. Jacobs, M.R. Sperling, K.A. Moxon, M.J. Kahana, J.J. Evans, and A.D. Sharan. Methods for implantation of micro-wire bundles and optimization of single/multiunit recordings from human mesial temporal lobe. J. Neural Eng. 11(2):026013, 2014.

14. Cogan, S.F. Neural Stimulation and Recording Electrodes. Annu. Rev. Biomed. Eng. 10:275-309, 2008.

15. Nordhausen, C.T., E.M. Maynard, and R.A. Normann. Single unit recording capabilities of a 100 microelectrode array. Brain Research. 726:129-140, 1996.

16. Simeral, J.D., S.P. Kim, M.J. Black, J.P. Donoghue, and L.R. Hochberg. Neural control of cursor trajectory and click by a human with tetraplegia 1000 days after implant of an intracortical microelectrode array. J. Neural Eng. 8(025027):1-25, 2011.

17. Nicolelis, M.A.L., A.A. Ghazanfar, B.M. Faggin, S. Votaw, and L.M.O. Oliveira. Reconstructing the Engram: Simultaneous Multisite, Manu Single Neuron Recordings. Neuron. 18:529-537, 1997.

18. Obaid, A., M.E. Hanna, Y.W. Wu, M. Kollo, R. Racz, M.R. Angle, J. Müller, N. Brackbill, W. Wray, F. Franke, E.J. Chichilnisky, A. Hierlemann, J.B. Ding, A.T. Schaefer, and N.A. Melosh. Massively parallel microwire arrays integrated with CMOS chips for neural recording. Science Advances. 6:1-10, 2020.

19. Wise, K.D., D.J. Anderson, J.F. Hetke, D.R. Kipke, and K. Najafi. Wireless Implantable Microsystems: High-Density Electronic Interfaces to the Nervous System. Proceedings of the IEEE. 92(1):76-97, 2004.

20. Kollo, M., R. Racz, M. Hanna, A. Obaid, M.R. Angle, W. Wray, Y. Kong, A. Hierlemann, J. Müller, N.A. Melosh, and A.T. Schaefer. CHIME: CMOS-hosted 
in-vivo microelectrodes for massively scalable neuronal recordings. bioRxiv. 2019.

21. Boergens, K.M., A. Tadić, M.S. Hopper, K. Sahasrabuddhe, Y. Kong, M. Straka, H.S. Sohal, and M.R. Angle. Laser Ablation of the Pia Mater for Insertion of High-Density Microelectrode Arrays in a Translational Sheep Model. bioRxiv, 2020.

22. Escamilla-MackertT., N.B. Langhals, T.D.Y. Kozai, and D.R. Kipke. Insertion of a three dimensional silicon microelectrode assembly through a thick meningeal membrane. IEEE Engineering in Medicine and Biology Society. Conference. 1616-1618, 2009.

23. John, S.E., T.J.H. Lovell, N.L. Opie, S. Wilson, T.C. Scordas, Y.T. Wong, G.S. Rind, S. Ronayne, S.H. Bauquier, C.N. May, D.B. Grayden, T.J. O'Brien, and T.J. Oxley. The ovine motor cortex: A review of functional mapping and cytoarchitecture. Neuroscience \& Behavioral Reviews. 80:306-315, 2017.

24. Weller, R.O., M.M. Sharp, M. Christodoulides, R.O. Carare, and K. Møllgård. The meninges as a barriers and facilitators for the movement of fluid, cells, and pathogens related to the rodent and human CNS. Acta. Neuropathologica. 135:363-385, 2018.

25. Kozai, T.D.Y., A.S. Jaquines-Gerstl, A.L. Vazquez, A.C. Michael, and X.T. Cui. Brain Tissue Resopnses to Neural Implants Impact Signal Sensitivity and Intervention Strategies. ACS Chem. Neurosci. 6:48-67, 2015.

26. Chen, R., A. Canales, and P. Anikeeva. Neural recording and modulation technologies. Nature Reviews. 2(16093):1-16, 2017.

27. Kralik, J.D., D.F. Dimitrov, D.J. Krupa, D.B. Katz, D. Cohen, and M.A.L. Nicolelis. Techniques for Chronic, Multisite Neuronal Ensemble Recordings in Behaving Animals. Methods. 25(2):121-150, 2001.

28. Rousche, P.J., and R.A. Normann. A method for pneumatically inserting an array of penetrating electrodes into cortical tissue. Annals of Biomedical Engineering. 20:413-422, 1992.

29. Seymour, J.P., and D.R. Kipke. Neural probe design for reduced tissue encapsulation in CNS. Biomaterial. 28:3594-3607, 2007.

30. Obidin, N., F. Tasmin, and C. Dagdeviren. The Future of Neuroimplantable Devices: A Materials Science and Regulatory Perspective. Advanced Materials. 1901482:1-26, 2019. 
31. Jun, J.J., N.A. Steinmetz, J.H. Siegle, D.J. Denman, M. Bauza, B. Barbarits, A.K. Lee, C.A. Anastassiou, A. Andrei, C. Aydin, M. Barbic, T.J. Blanche, V. Bonin, J. Couto, B. Dutta, S.L. Gratiy, D.A. Gutnisk, M. Hausser, B. Karsh, P. Ledochowitsch, C.M. Lopez, C. Mitelut, S. Musa, M. Okun, M. Pachitarius, J. Putzeys, P.D. Rich, C. Rossant, W.L. Sun, K. Svoboda, M. Carandini, and K.D. Harris. Fully integrated silicon probes for high-density recording of neural activity. Nature. 331:232-236, 2017.

32. Pandarinath, C., P. Nuyujukian, C.H. Blabe, B.L. Sorice, J. Saab, F.R. Willett, L.R. Hochberg, K.V. Shenoy, and J.M. Henderson. High performance communication by people with paralysis using an intracortical brain-computer interface. Elife. 6:e18554, 2017.

33. Flesher, S.N., J.L. Collinger, S.T. Foldes, J.M. Weiss, J.E. Downey, E.C. TylerKabara, S.J. Bensmaia, A.B. Schwartz, M.L. Boninger, and R.A. Gaunt. Intracortical microstimulation of human somatosensory cortex. Sci Transl Med. 8(361):361ra141, 2016.

34. Ehrlich, Paul. Dielectric Properties of Teflon from Room Temperature to $314{ }^{\circ} \mathrm{C}$ and from Frequencies of $10^{2}$ to $10^{5} \mathrm{c} / \mathrm{s}$. Journal of Research of the National Bureau of Standards. 51(4):185-188, 1953.

35. FDA. Metals Used in Medical Devices, 2020. at https://www.fda.gov/medicaldevices/products-and-medical-procedures/metals-used-medical-devices.

36. Ribeiro, A.M., T.H.S. Flores-Sahagun, and R.C. Paredes. A perspective on molybdenum biocompatibility and antimicrobial activity for applications in implants. Journal of Materials Science. 51:2806-2816, 2016.

37. Kahouli, A., A. Sylvestre, J-F Laithier, S. Pairis, J-L Garden, E. Andre, F. Jomni, and $\mathrm{B}$. Yangui. Effect of $\mathrm{O}_{2}, \mathrm{Ar} / \mathrm{H}_{2}$ and $\mathrm{CF}_{4}$ plasma treatments on the structural and dielectric properties of parylene-C thin films. J. Phys. D: Appl. Phys. 45:215306, 2012.

38. Material Property Database. Polyimide, at http://www.mit.edu/ 6.777/matprops/polyimide.htm

39. Westphal, W.B. and A. Sils. Dielectric Constant and Loss Data. Technical Report AFML-TR-72-39. 1972.

40. HajjHassan, M., V. Chodavarapu, and S. Musallam. NeuroMEMS: Neural Probe Microtechnologies. Sensors. 8:6704-6726, 2008. 
41. Fine, Ione. Bootstrapping Examples, 2015. at https://courses.washington.edu/matlab1/Bootstrap_examples.html

42. Faul, F., E. Erdfelder, A.G. Lang, and A. Buchner. G*Power 3: A flexible statistical power analysis program for the social, behavioral, and biomedical sciences. Behavior Research Methods. 39:175-191, 2007.

43. Faul, F., E. Erdfelder, A. Buchner, and A.G. Lang. Statistical power analyses using G*Power 3.1: Tests for correlation and regression analyses. Behavior Research Methods. 41:1149-1160. 
APPENDIX A: RAW PERCENTAGE CONNECTIVITY DATA

\begin{tabular}{|c|c|c|c|c|}
\hline Diameter (mm) & Pitch $(\mu \mathrm{m})$ & $\begin{array}{c}\text { Total } \\
\text { Microwires }\end{array}$ & $\begin{array}{l}\text { Connected } \\
\text { Microwires }\end{array}$ & $\begin{array}{c}\text { Percentage } \\
\text { Connectivity } \\
(\%)\end{array}$ \\
\hline 3 & 200 & 274 & 211 & 77.01 \\
\hline 3 & 200 & 251 & 184 & 73.31 \\
\hline 3 & 200 & 253 & 162 & 64.03 \\
\hline 3 & 200 & 253 & 125 & 49.41 \\
\hline 3 & 200 & 189 & 165 & 87.30 \\
\hline 3 & 200 & 253 & 157 & 62.06 \\
\hline 3 & 200 & 253 & 192 & 75.89 \\
\hline 3 & 200 & 253 & 84 & 33.20 \\
\hline 3 & 100 & 700 & 441 & 63.00 \\
\hline 3 & 100 & 700 & 319 & 45.57 \\
\hline 12 & 200 & 2500 & 1773 & 70.92 \\
\hline 12 & 200 & 2500 & 2128 & 85.12 \\
\hline 12 & 200 & 2500 & 2085 & 83.40 \\
\hline 12 & 200 & 2500 & 2071 & 82.84 \\
\hline 12 & 200 & 2500 & 1950 & 78.00 \\
\hline 12 & 200 & 2500 & 1791 & 71.64 \\
\hline 12 & 100 & 10000 & 9792 & 97.92 \\
\hline 12 & 100 & 10000 & 5307 & 53.07 \\
\hline 12 & 100 & 10000 & 6702 & 67.02 \\
\hline 12 & 100 & 10000 & 8518 & 85.18 \\
\hline 12 & 100 & 10000 & 4265 & 42.65 \\
\hline
\end{tabular}

